\title{
Inter-specific competition among trees in pythagorean fuzzy soft environment
}

\author{
Muhammad Akram ${ }^{1}$ (1) $\cdot$ Hafiza Saba Nawaz ${ }^{1}$
}

Received: 10 January 2021 / Accepted: 10 July 2021 / Published online: 10 August 2021

(c) The Author(s) 2021

\begin{abstract}
A Pythagorean fuzzy set is very effective mathematical framework to represent parameter-wise imprecision which is the property of linguistic communication. A Pythagorean fuzzy soft graph is more potent than the intuitionistic fuzzy soft as well as the fuzzy soft graph as it depicts the interactions among the objects of a system using Pythagorean membership grades with respect to different parameters. This article addresses the content of competition graphs as well as economic competition graphs like $k$-competition graphs, $m$-step competition graphs and $p$-competition graphs in Pythagorean fuzzy soft environment. All these concepts are illustrated with examples and fascinating results. Furthermore, an application which describes the competition among distinct forest trees, that grow together in the mixed conifer forests of California, for plant resources is elaborated graphically. An algorithm is also designed for the construction of Pythagorean fuzzy soft competition graphs. It is worthwhile to express the competing and non-competing interactions in various networks with the help of Pythagorean fuzzy soft competition graphs wherein a variation in competition relative to different attributes is visible.
\end{abstract}

Keywords Competition graphs $\cdot$ Economic competition graphs $\cdot$ Competition among plants

\section{Introduction}

A graph is an effective tool to depict the connectivity and relationship among the objects of a system. For all the straightforward as well as complex networks can be modeled in terms of graph. These networks include the telecommunication web in computer sciences, molecular structures of organic compounds in chemistry, electronic circuits of physics, road networks in transportation engineering, food webs in ecology and cellular dynamics in biology. Thus, all pairwise real-world relationships can be illustrated with the help of graphs. The remarkable work of Swiss mathematician Euler, in 1736, for the Königsberg problem of seven bridges is considered to be the formal commencement of graph theory. Afterwards, these mathematical structures were not only applied in other disciplines but also emerged as an independent subject.

Muhammad Akram

m.akram@pucit.edu.pk

Hafiza Saba Nawaz

sabanawaz707@gmail.com

1 Department of Mathematics, University of the Punjab, New Campus, Lahore 54590, Pakistan
A graph in which a direction is associated with every edge is called directed graph. Cohen [7] considered the model of food web as a directed graph and stated that two species (vertices) are joined by edge in the corresponding competition graph (CG), if these species must have at least one prey in common and vice versa. These CGs were then applied in various systems to depict the competition among the constituents of that system. Various authors also studied different forms of CGs like $p$-CGs [14], tolerance CGs [4], $m$-step CGs [6] and competition hypergraphs [33]. Different examples of such systems are competition among sellers for buyers in imperfect markets, among the applicants for a post, among animals for food and shelter, among plants for resources and many more. Since all these systems have uncertainty in their data, therefore it is better to handle them with fuzzy set (FS) theory and its extensions.

The FS [36] was suggested by Zadeh in 1965 as an extension of crisp set to deal with uncertainty. It is a function $\mu$ that takes elements of universal set $X$ as an input and maps on the members of unit closed interval, that is, $\mu: X \rightarrow[0,1]$. The value assigned to the element $x \in X$ in FS is called the membership grade $\mu(x)$ of that element $x$ and illustrates the extent of belongingness of $x$ in this set. So FS represents the evidence for $x \in X$, but it lacks the ability to represent the 
evidence against $x \in X$. To address this issue, Atanassov [3], in 1983, put forward the intuitionistic fuzzy set (IFS) by inserting a new component $v: X \rightarrow[0,1]$ that investigates the non-membership grade such that $\mu+v \leq 1$. In this way, IFS provides more flexibility as compared to FS theory due its non-null hesitation part. Yager [35] introduced another non-standard fuzzy subset, the Pythagorean fuzzy set (PFS), by relating the negation operation with Pythagorean theorem. This set assigns the membership and non-membership values so that $\mu^{2}+v^{2} \leq 1$. He also compared the IFS and PFS and declared that the set of intuitionistic membership values is smaller than that of Pythagorean membership grades. All these extensions of crisp sets dealt with many actual-world problems that have fuzziness and uncertainty.

Another form of uncertainty was indicated by Molodtsov [21] in 1999. He gave the idea of soft set $\left(\mathrm{S}_{f} \mathrm{~S}\right)$ which is a parameterized collection of subsets of universe $X$. It is defined as a set-valued approximate mapping from a set of parameters (or attributes which are selected according to the members of $X$ ) to the power set of $X$. This set was applied to various systems by different authors [5-19]. In order to add the flavor of fuzziness to $S_{f} S$, Maji et al. [16] put forward the fuzzy soft set and also presented its properties. This hybrid model contributed to the solution of many decision making problems highlighted by Roy and Maji [28]. Maji et al. [17] introduced the intuitionistic fuzzy soft set and Peng et al. [27] put forth Pythagorean fuzzy soft sets ( $\left.\mathrm{PFS}_{f} \mathrm{Ss}\right)$. These hybrid models have tremendous applications in various fields. Garg [8-11] considered Pythagorean fuzzy sets with applications in multiple criteria decision-making.

Different graphs were studied in FS theory and its related extensions later on. Kaufmann [13] presented fuzzy graph and its operations were defined by Mordeson and Nair [22]. Paravathi and Karunambigai [26] put forward intuitionistic fuzzy graphs with suitable illustrations to its idea. For the implication of Pythagorean membership grades in graphs, Naz et al. [25] introduced the Pythagorean fuzzy graphs (PFGs). They also determined various properties of these graphs, Pythagorean fuzzy preference relations as well as applications to some decision-making problems modeled in Pythagorean fuzzy environment. Mordeson et al. [23] discussed many fuzzy graph theoretic problems regarding to human trafficking.

Soft graph which is based on the structure of $\mathbf{S}_{f} \mathrm{~S}$ is, in fact, a parameterized family of graphs and was suggested by Thumbakara and George [34]. Akram and Nawaz [1] combined the concept of fuzzy graphs and soft graph to put forward a new notion of fuzzy soft graphs. For the involvement of Pythagorean membership grades to the idea of soft graph, Akram and Shahzadi [32] introduced the Pythagorean fuzzy soft graph $\left(\mathrm{PFS}_{f} \mathrm{G}\right)$ and discussed its regularity.

With the passage of time, the concept of CGs was also elaborated in different theories. Samanta and Pal [31] pro- posed fuzzy CGs and some of its generalizations. The $m$-step fuzzy CGs [30], intuitionistic fuzzy CGs [29], q-rung orthopair fuzzy CGs [12], q-rung picture fuzzy CGs [2] and fuzzy soft CGs [24] were suggested by different authors. All these research articles include various interesting applications of CGs in social, ecological as well as economic competing network models.

There exist various competing networks that exhibit variation with respect to distinct attributes. To show the competing entities of such systems, it is necessary to include the parametrization factor to Pythagorean fuzzy competition graphs (PFCGs). The motivation of this paper is the following:

1. The $\mathrm{PFS}_{f} \mathrm{~S}$ is a hybrid model of PFS and $\mathrm{S}_{f} \mathrm{~S}$, and has the combined properties of both these ground sets. The $\mathrm{PFS}_{f}$ Gs are able to represent all those interactions that have Pythagorean membership grades and depend on different factors.

2. The introduction to Pythagorean fuzzy soft competition graphs (PFS ${ }_{f} \mathrm{CGs}$ ) and Pythagorean fuzzy soft economic competition graphs (PFS ${ }_{f}$ ECGs) is essential to depict the competition in the ecological as well as economic networks, respectively, of $\mathrm{PFS}_{f}$ Gs so that different measures can be taken in different situations to minimize that competition.

This article contributes to the existing literature as follows:

1. It first defines some important definitions related to $\mathrm{PFS}_{f}$ Ss like height, cardinality and support of these sets which are used in further research work.

2. It updates the readers with new concept of $\mathrm{PFS}_{f} \mathrm{CGs}$, PFS $_{f}$ ECGs and various other definitions as well as important results.

3. It provides an example of competing forest trees for the resources in $\mathrm{PFS}_{f}$ environment, where quantity of the resources that are a cause of growth promotion as well as inhibition of trees vary with seasonal changes.

4. It renders an algorithm to evaluate the strength of competing and non-competing interaction of parameterdependent competing network.

The layout of this article is as follows. The next section gives preliminary concepts. The PFS ${ }_{f}$ CGs and PFS ${ }_{f}$ ECGs and other related definitions are provided in the next section. All these definitions are also elaborated with the help of examples and explained with the help of theorems. In the following section, we have used the proposed concept of CGs in $\mathrm{PFS}_{f} \mathrm{~S}$ theory to represent the competition among forest trees of California. The plants require nutrients, water, sunlight, etc. for their growth. When the concentrations of these plant resources are suboptimal or not equally dis- 
Table 1 List of acronyms

\begin{tabular}{ll}
\hline Acronyms & Description \\
\hline FS & Fuzzy set \\
IFS & Intuitionistic Fuzzy set \\
PFS & Pythagorean fuzzy set \\
S $_{f} \mathrm{~S}$ & Soft set \\
PFS $_{f} \mathrm{~S}$ & Pythagorean fuzzy soft set \\
PFG $_{\text {PFS }} \mathrm{G}$ & Pythagorean fuzzy graph \\
$\mathrm{CG}$ & Pythagorean fuzzy soft graph \\
PFCG $_{\text {PFECG }}$ & Competition graph \\
PFS $_{f}$ CG & Pythagorean fuzzy competition graph \\
PFS $_{f}$ ECG & Pythagorean fuzzy economic competition graph \\
\hline
\end{tabular}

tributed, they compete with one another for these resources in order to survive. The availability of these natural resources vary from season to season so the discussion of competition in $\mathrm{PFS}_{f}$ environment permits to examine competition with respect to different parameters which prevent the information loss. Moreover, the assignment of Pythagorean membership grades to the constituents of this system provide us information about both the involvement and non-involvement of trees in competition. This competition is also presented graphically. The comparative analysis is given in the following section and the concluding section completes the article. The list of acronyms is provided in Table 1.

\section{Preliminaries}

Throughout this section, we will consider $X$ as a non-empty universal set, $P$ as the set of parameters regarding to all objects in the universe and $Q$ as a subset of $P$ :

Definition 1 [35] A PFS A over the universal set $X$ is defined as an object $A=\left(\mu_{A}, v_{A}\right): X \rightarrow[0,1] \times[0,1]$, where $\mu_{A}:$ $X \rightarrow[0,1]$ and $v_{A}: X \rightarrow[0,1]$ represent the membership and non-membership functions, respectively, such that $0 \leq$ $\mu_{A}^{2}(x)+v_{A}^{2}(x) \leq 1$ holds for all $x \in X$. The set notation of PFS $A$ is $A=\left\{\left\langle x,\left(\mu_{A}(x), v_{A}(x)\right)\right\rangle \mid x \in X\right\}$, and $\pi_{A}(x)=$ $\sqrt{1-\mu_{A}^{2}(x)-v_{A}^{2}(x)}$ is the Pythagorean fuzzy index of the element $x$ to the set $A$.

Definition 2 [27] $\mathrm{A} \mathrm{PFS}_{f} \mathrm{~S}(\mathbb{A}, Q)$ over $X$ is defined by the Pythagorean fuzzy approximation function $\mathbb{A}: Q \rightarrow$ $P(X)$, where $P(X)$ denotes the Pythagorean fuzzy power set over $X$ containing all possible PFSs over $X$. Its set notation is is follows: $\mathbb{A}=\{\langle\mathfrak{q}, \mathbb{A}(\mathfrak{q})\rangle \mid \forall \mathfrak{q} \in Q\}$, where $\mathbb{A}(\mathfrak{q})=\left\{\left\langle x,\left(\mu_{\mathbb{A}(\mathfrak{q})}(x), v_{\mathbb{A}(\mathfrak{q})}(x)\right)\right\rangle \mid \forall x \in X\right\}$ with $0 \leq$
$\mu_{\AA}^{2}(\mathfrak{q})(x)+v_{\mathbb{A}(\mathfrak{q})}^{2}(x) \leq 1$ for all $x \in X$, is a PFS relative to parameter $\mathfrak{q}$.

Definition 3 [32] A PFS $f \mathrm{G} \mathbb{G}$ on $X$ is an ordered 3-tuple $\mathbb{G}=(\mathbb{A}, \mathbb{B}, Q)$ such that

(i) $Q$ denotes a non-empty set of attributes,

(ii) $(\mathbb{A}, Q)$ represents a $\mathrm{PFS}_{f} \mathrm{~S}$ over $X$, i.e., $\mathbb{A}: Q \rightarrow$ $P(X)$,

(iii) $(\mathbb{B}, Q)$ represents a $\mathrm{PFS}_{f}$ relation on $X$, i.e., $\mathbb{B}: Q \rightarrow$ $P(X \times X)$,

(iv) $\mathbb{R}(\mathfrak{q})=(\mathbb{A}(\mathfrak{q}), \mathbb{B}(\mathfrak{q}))$ is a PFG, for all $\mathfrak{q} \in Q$.

That is, the membership and non-membership values of the Pythagorean fuzzy edge $(x, y)$ in $\mathbb{R}(\mathfrak{q})$ is given by

$\left\{\begin{array}{l}\mu_{\mathbb{B}(\mathfrak{q})}(x, y) \leq \min \left\{\mu_{\mathbb{A}(\mathfrak{q})}(x), \mu_{\mathbb{A}(\mathfrak{q})}(y)\right\}, \\ v_{\mathbb{B}(\mathfrak{q})}(x, y) \leq \max \left\{v_{\mathbb{A}(\mathfrak{q})}(x), v_{\mathbb{A}(\mathfrak{q})}(y)\right\},\end{array}\right.$

respectively, where $0 \leq \mu_{\mathbb{B}(\mathfrak{q})}^{2}(x, y)+v_{\mathbb{B}(\mathfrak{q})}^{2}(x, y) \leq 1$ holds for all $\mathfrak{q} \in Q$ and $x, y \in X$.

$(\mathbb{A}, Q)$ is called $\mathrm{PFS}_{f}$ vertex set and $(\mathbb{B}, Q)$ is called $\mathrm{PFS}_{f}$ edge set for the $\mathrm{PFS}_{f} \mathrm{G} \mathbb{G}$. Moreover, the element $x$ in $(\mathbb{A}, Q)$ is termed as $\operatorname{PFS}_{f}$ vertex and $(x, y)$ in $(\mathbb{B}, Q)$ is known as $\mathrm{PFS}_{f}$ edge.

Definition 4 We define support $\operatorname{supp}(\mathbb{A}, Q)$ of a $\mathrm{PFS}_{f} \mathrm{~S}$ $(\mathbb{A}, Q)$ as $\operatorname{supp}(\mathbb{A}, Q)=\left\{\left\langle\mathfrak{q}, \operatorname{supp}_{\mu}(\mathbb{A}(\mathfrak{q})) \cup \operatorname{supp}_{v}(\mathbb{A}(\mathfrak{q}))\right\rangle\right\}$, where $\operatorname{supp}_{\mu}(\mathbb{A}(\mathfrak{q}))=\left\{x \in X \mid \mu_{\mathbb{A}(\mathfrak{q})}(x)>0\right\}$ and $\operatorname{supp}_{v}(\mathbb{A}(\mathfrak{q}))=\left\{x \in X \mid v_{\mathbb{A}(\mathfrak{q})}(x)<1\right\}$, for each $\mathfrak{q} \in Q$.

Definition 5 We define cardinality $\operatorname{Card}(\mathbb{A}, Q)$ of a $\mathrm{PFS}_{f} \mathrm{~S}$ $(\mathbb{A}, Q)$ as $\operatorname{Card}(\mathbb{A}, Q)=\sum_{\mathfrak{q} \in Q} \operatorname{Card}(\mathbb{A}(\mathfrak{q}))$, where $\operatorname{Card}(\mathbb{A}(\mathfrak{q}))=\left(|\mathbb{A}(\mathfrak{q})|_{\mu},|\mathbb{A}(\mathfrak{q})|_{\nu}\right)$ such that $|\mathbb{A}(\mathfrak{q})|_{\mu}=$ $\sum_{x \in X} \mu_{\mathbb{A}(\mathfrak{q})}(x)$ and $|\mathbb{A}(\mathfrak{q})|_{\nu}=\sum_{x \in X} v_{\mathbb{A}(\mathfrak{q})}(x)$, for each $\mathfrak{q} \in Q$.

Definition 6 We define height $h(\mathbb{A}, Q)$ of a $\operatorname{PFS}_{f} \mathrm{~S}(\mathbb{A}, Q)$ as $h(\mathbb{A}, Q)=\left(\sup _{\mathfrak{q} \in Q} h_{\mu}(\mathbb{A}(\mathfrak{q})), \inf _{\mathfrak{q} \in Q} h_{\nu}(\mathbb{A}(\mathfrak{q}))\right)$, where $h_{\mu}(\mathbb{A}(\mathfrak{q}))=\sup _{x \in X}\left(\mu_{\mathbb{A}(\mathfrak{q})}(x)\right)$ and $h_{v}(\mathbb{A}(\mathfrak{q}))=\inf _{x \in X}$ $\left(v_{\mathbb{A}(\mathfrak{q})}(x)\right)$, for each $\mathfrak{q} \in Q$.

Definition 7 The strength $\operatorname{Str}(x, y)$ of a $\mathrm{PFS}_{f}$ edge $(x, y)$ in the $\operatorname{PFS}_{f} \mathrm{G} \mathbb{G}=(\mathbb{A}, \mathbb{B}, Q)$ is defined as $\operatorname{Str}(x, y)=$ $\left(\operatorname{Str}(x, y)_{\mu}, \operatorname{Str}(x, y)_{v}\right)=\left(\min _{\mathfrak{q}}\left\{\operatorname{Str}(\mathfrak{q} ;(x, y))_{\mu}\right\}, \max _{\mathfrak{q}}\right.$ $\left.\left\{\operatorname{Str}(\mathfrak{q} ;(x, y))_{\nu}\right\}\right)$, where

$\left\{\begin{array}{l}\operatorname{Str}(\mathfrak{q} ;(x, y))_{\mu}=\frac{\mu_{\mathbb{B}(\mathfrak{q})}(x, y)}{\mu_{\mathbb{A}(\mathfrak{q})}(x) \wedge \mu_{\mathbb{A}(\mathfrak{q})}(y)}, \\ \operatorname{Str}(\mathfrak{q} ;(x, y))_{\nu}=\frac{v_{\mathbb{B}(\mathfrak{q})}(x, y)}{v_{\mathbb{A}(\mathfrak{q})}(x) \vee v_{\mathbb{A}(\mathfrak{q})}(y)} .\end{array}\right.$

Note that $\left(\operatorname{Str}(\mathfrak{q} ;(x, y))_{\mu}, \operatorname{Str}(\mathfrak{q} ;(x, y))_{\nu}\right)$ denotes the strength of Pythagorean fuzzy edge $(x, y)$ in the PFG $\mathbb{R}(\mathfrak{q})=$ $(\mathbb{A}(\mathfrak{q}), \mathbb{B}(\mathfrak{q}))$. 
Fig. 1 A PFS $f$ G with strong and weak edges

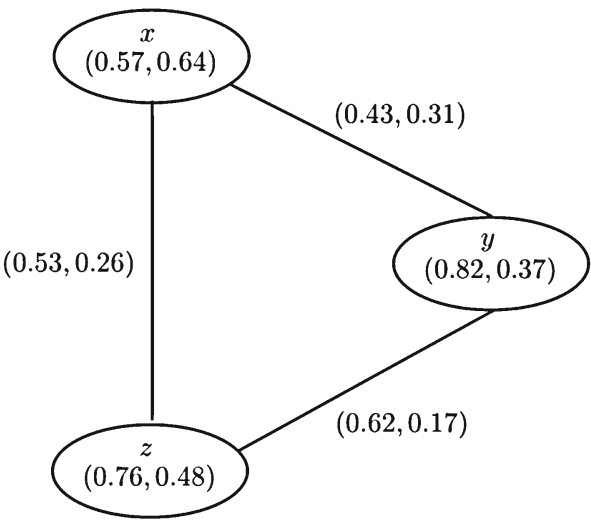

$R\left(q_{1}\right)$

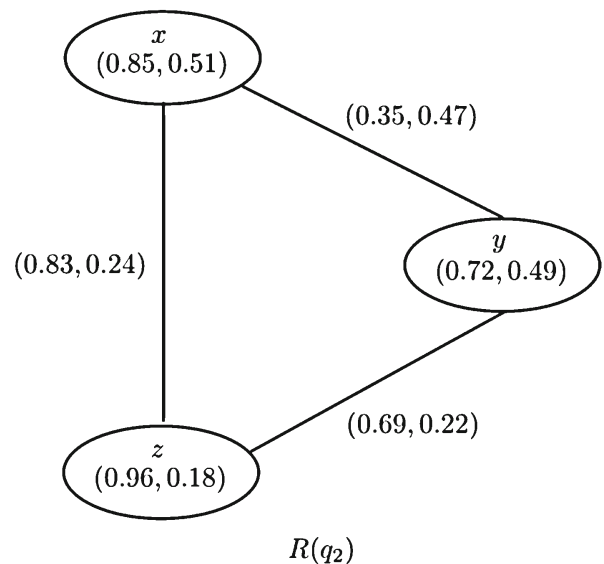

Definition $8 \mathrm{APFS}_{f}$ edge $(x, y)$ is called independent strong in $\mathbb{G}=(\mathbb{A}, \mathbb{B}, Q)$ if the Pythagorean fuzzy edge $(x, y)$ is independent strong in PFG $\mathbb{R}(\mathfrak{q})=(\mathbb{A}(\mathfrak{q}), \mathbb{B}(\mathfrak{q}))$ for every $\mathfrak{q}$, i.e., following inequalities hold for all $\mathfrak{q}$ :

$\left\{\begin{array}{l}\mu_{\mathbb{B}(\mathfrak{q})}(x, y) \geq \frac{1}{2}\left(\mu_{\mathbb{A}(\mathfrak{q})}(x) \wedge \mu_{\mathbb{A}(\mathfrak{q})}(y)\right), \\ v_{\mathbb{B}(\mathfrak{q})}(x, y) \leq \frac{1}{2}\left(v_{\mathbb{A}(\mathfrak{q})}(x) \vee v_{\mathbb{A}(\mathfrak{q})}(y)\right) .\end{array}\right.$

The edge $(x, y)$ is called weak if above-mentioned conditions are not satisfied for some $\mathfrak{q}$.

In other words, a $\operatorname{PFS}_{f}$ edge $(x, y)$ is called independent strong in $\mathbb{G}=(\mathbb{A}, \mathbb{B}, Q)$ if $\operatorname{Str}(x, y)_{\mu} \geq 0.5$ and $\operatorname{Str}(x, y)_{v} \leq 0.5$, and weak if not.

Example 1 Let us consider a $\operatorname{PFS}_{f} \mathrm{G} \mathbb{G}=(\mathbb{A}, \mathbb{B}, Q)$ displayed in Fig. 1.

Since $\operatorname{Str}(x, y)=(0.48,0.92), \operatorname{Str}(x, z)=(0.82,0.45)$ and $\operatorname{Str}(y, z)=(0.92,0.47)$, therefore, the $\operatorname{PFS}_{f}$ edges $(x, z)$ and $(y, z)$ are independent strong, whereas $(x, y)$ is weak in the $\operatorname{PFS}_{f} \mathrm{G} \mathbb{G}$.

Definition $9 \mathrm{~A} \mathrm{PFS}_{f} \mathrm{G} \mathbb{G}^{\prime}=\left(\mathbb{A}^{\prime}, \mathbb{B}^{\prime}, Q\right)$ is called a PFS $f$ subgraph of $\mathbb{G}=(\mathbb{A}, \mathbb{B}, Q)$ if $\mathbb{A}^{\prime} \leq \mathbb{A}$ ( similarly, $\mathbb{B}^{\prime} \leq$ $\mathbb{B})$, i.e., $\mu_{\mathbb{A}^{\prime}(\mathfrak{q})}(x) \leq \mu_{\mathbb{A}(\mathfrak{q})}(x)\left(\right.$ similarly, $\mu_{\mathbb{B}^{\prime}(\mathfrak{q})}(x, y) \leq$ $\left.\mu_{\mathbb{B}(\mathfrak{q})}(x, y)\right)$ and $v_{\mathbb{A}^{\prime}(\mathfrak{q})}(x) \geq v_{\mathbb{A}(\mathfrak{q})}(x)\left(\operatorname{similarly}, v_{\mathbb{B}^{\prime}(\mathfrak{q})}(x, y)\right.$ $\left.\geq v_{\mathbb{B}(\mathfrak{q})}(x, y)\right)$ for each $x, y \in X$ and $\mathfrak{q} \in Q$.

Definition $10 \mathrm{~A} \mathrm{PFS}_{f}$ digraph $\overrightarrow{\mathbb{G}}$ on universal set $X$ is an ordered 3-tuple $\overrightarrow{\mathbb{G}}=(\mathbb{A}, \vec{B}, Q)$ such that

(i) $Q$ is a non-void set of attributes,

(ii) $(\mathbb{A}, Q)$ is a $\operatorname{PFS}_{f}$ S over $X$, i.e., $\mathbb{A}: Q \rightarrow P(X)$,

(iii) $(\overrightarrow{\mathbb{B}}, Q)$ is a $\mathrm{PFS}_{f}$ relation on $X$, i.e., $\overrightarrow{\mathbb{B}}: Q \rightarrow P(X \times$

(iv) $\stackrel{X}{\overrightarrow{\mathbb{R}}}(\mathfrak{q})=(\mathbb{A}(\mathfrak{q}), \overrightarrow{\mathbb{B}}(\mathfrak{q}))$ is a Pythagorean fuzzy digraph, for all $\mathfrak{q} \in Q$.
That is, the membership and non-membership degrees of a Pythagorean fuzzy arc $(x, y)$ in $\overrightarrow{\mathbb{R}}(\mathfrak{q})$ is given by

$\left\{\begin{array}{l}\mu_{\overrightarrow{\mathbb{B}}(\mathfrak{q})}(x, y) \leq \min \left\{\mu_{\mathbb{A}(\mathfrak{q})}(x), \mu_{\mathbb{A}(\mathfrak{q})}(y)\right\}, \\ \nu_{\overrightarrow{\mathbb{B}}(\mathfrak{q})}(x, y) \leq \max \left\{\nu_{\mathbb{A}(\mathfrak{q})}(x), \nu_{\mathbb{A}(\mathfrak{q})}(y)\right\},\end{array}\right.$

for each $\mathfrak{q}$ and $x, y \in X$, such that the condition $0 \leq$ $\mu_{\overrightarrow{\mathbb{B}}(\mathfrak{q})}^{2}(x, y)+\nu_{\overrightarrow{\mathbb{B}}(\mathfrak{q})}^{2}(x, y) \leq 1$ must holds. Moreover, $(\mathbb{A}, Q)$ is $\mathrm{PFS}_{f}$ vertex set and $(\overrightarrow{\mathbb{B}}, Q)$ is $\mathrm{PFS}_{f}$ edge set for the $\operatorname{PFS}_{f}$ digraph $\overrightarrow{\mathbb{G}}$.

\section{Pythagorean fuzzy soft competition graphs}

Definition 11 The PFS $f$ out-neighborhood $\mathbb{N}^{+}(x)$ of a vertex $x$ in $\operatorname{PFS}_{f}$ digraph $\overrightarrow{\mathbb{G}}=(\mathbb{A}, \overrightarrow{\mathbb{B}}, Q)$ is a $\operatorname{PFS}_{f} \mathrm{~S}$ $\mathbb{N}^{+}(x)=\left\{\left\langle\mathfrak{q}, \mathbb{N}^{+}(\mathfrak{q})(x)\right\rangle \mid \forall \mathfrak{q} \in Q\right\}$, where $\mathbb{N}^{+}(\mathfrak{q})(x)=$ $\left\{\left\langle y,\left(\mu_{\overrightarrow{\mathbb{B}}(\mathfrak{q})}(x, y), \nu_{\overrightarrow{\mathbb{B}}(\mathfrak{q})}(x, y)\right)\right\rangle \mid \mu_{\vec{B}_{(\mathfrak{q})}}(x, y)>0\right.$ or $\nu_{\overrightarrow{\mathbb{B}}(\mathfrak{q})}$ $(x, y)>0\}$. The $\mathrm{PFS}_{f}$ in-neighborhood $\mathbb{N}^{-}(x)$ of a vertex $x$ in $\operatorname{PFS}_{f}$ digraph $\overrightarrow{\mathbb{G}}=(\mathbb{A}, \overrightarrow{\mathbb{B}}, Q)$ is a $\operatorname{PFS}_{f} \mathrm{~S}$ $\mathbb{N}^{-}(x)=\left\{\left\langle\mathfrak{q}, \mathbb{N}^{-}(\mathfrak{q})(x)\right\rangle \mid \forall \mathfrak{q} \in Q\right\}$, where $\mathbb{N}^{-}(\mathfrak{q})(x)=$ $\left\{\left\langle y,\left(\mu_{\mathbb{B}_{(\mathfrak{q})}}(y, x), \nu_{\vec{B}_{(\mathfrak{q})}}(y, x)\right)\right\rangle \mid \mu_{\vec{B}_{(\mathfrak{q})}}(y, x)>0\right.$ or $v_{\overrightarrow{\mathbb{B}}(\mathfrak{q})}$ $(y, x)>0\}$.

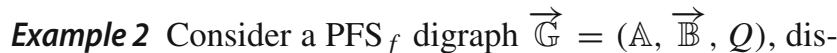
played in Fig. 2, such that $\overrightarrow{\mathbb{G}}=\left(\overrightarrow{\mathbb{R}}\left(\mathfrak{q}_{1}\right), \overrightarrow{\mathbb{R}}\left(\mathfrak{q}_{2}\right)\right)$, where

$$
\begin{aligned}
\overrightarrow{\mathbb{R}}\left(\mathfrak{q}_{1}\right)= & \left(\mathbb{A}\left(\mathfrak{q}_{1}\right), \overrightarrow{\mathbb{B}}\left(\mathfrak{q}_{1}\right)\right)=(\{\langle v,(0.59,0.65)\rangle, \\
& \langle w,(0.74,0.42)\rangle,\langle x,(0.82,0.36)\rangle, \\
& \langle y,(0.68,0.58)\rangle,\langle z,(0.34,0.89)\rangle\}, \\
& \langle((v, w),(0.51,0.62)\rangle,\langle(v, x),(0.54,0.64)\rangle, \\
& \langle(v, y),(0.55,0.47)\rangle, \\
& \langle(y, w),(0.66,0.57)\rangle,\langle(y, x),(0.63,0.49)\rangle, \\
& \langle(y, z),(0.31,0.82)\rangle,\langle(z, w),(0.28,0.78)\rangle\}),
\end{aligned}
$$




$$
\begin{aligned}
\overrightarrow{\mathbb{R}}\left(\mathfrak{q}_{2}\right)= & \left(\mathbb{A}\left(\mathfrak{q}_{2}\right), \overrightarrow{\mathbb{B}}\left(\mathfrak{q}_{2}\right)\right)=(\{\langle v,(0.64,0.54)\rangle, \\
& \langle w,(0.84,0.32)\rangle,\langle x,(0.75,0.66)\rangle, \\
& \langle y,(0.39,0.46)\rangle,\langle z,(0.58,0.79)\rangle\}, \\
\{ & \langle(w, v),(0.61,0.49)\rangle,\langle(w, x),(0.72,0.51)\rangle, \\
& \langle(w, z),(0.57,0.72)\rangle,\langle(x, v),(0.63,0.48)\rangle, \\
& \langle(x, y),(0.38,0.55)\rangle, \\
& \langle(z, v),(0.59,0.42)\rangle,\langle(z, x),(0.58,0.73)\rangle, \\
& \langle(z, y),(0.33,0.69)\rangle\}) .
\end{aligned}
$$

The $\mathrm{PFS}_{f}$ out-neighborhoods of $\mathrm{PFS}_{f}$ vertices of $\overrightarrow{\mathbb{G}}$ are

$$
\left\{\begin{aligned}
\mu_{\mathbb{D}(\mathfrak{q})}(x, y)= & \left(\mu_{\mathbb{A}(\mathfrak{q})}(x) \wedge \mu_{\mathbb{A}(\mathfrak{q})}(y)\right) h_{\mu}\left(\mathbb{N}^{+}(\mathfrak{q})(x)\right. \\
& \left.\cap \mathbb{N}^{+}(\mathfrak{q})(y)\right) \\
v_{\mathbb{D}(\mathfrak{q})}(x, y)= & \left(v_{\mathbb{A}(\mathfrak{q})}(x) \vee v_{\mathbb{A}(\mathfrak{q})}(y)\right) h_{\nu}\left(\mathbb{N}^{+}(\mathfrak{q})(x)\right. \\
& \left.\cap \mathbb{N}^{+}(\mathfrak{q})(y)\right),
\end{aligned}\right.
$$

such that $0 \leq \mu_{\mathbb{D}(\mathfrak{q})}^{2}(x, y)+v_{\mathbb{D}(\mathfrak{q})}^{2}(x, y) \leq 1$, for all $x, y \in X$ and $\mathfrak{q} \in Q$.

Example 3 Consider the $\operatorname{PFS}_{f} \mathrm{G} \overrightarrow{\mathbb{G}}=(\mathbb{A}, \overrightarrow{\mathbb{B}}, Q)$ of Example 2.

$$
\begin{aligned}
\mathbb{N}^{+}(v) & =\left\{\frac{\{\langle w,(0.51,0.62)\rangle,\langle x,(0.54,0.64)\rangle,\langle y,(0.55,0.47)\rangle\}}{\mathfrak{q}_{1}}, \frac{\{\}}{\mathfrak{q}_{2}}\right\}, \\
\mathbb{N}^{+}(w) & =\left\{\frac{\{\}}{\mathfrak{q}_{1}}, \frac{\{\langle v,(0.61,0.49)\rangle,\langle x,(0.72,0.51)\rangle,\langle z,(0.57,0.72)\}}{\mathfrak{q}_{2}}\right\}, \\
\mathbb{N}^{+}(x) & =\left\{\frac{\{\}}{\mathfrak{q}_{1}}, \frac{\{\langle v,(0.63,0.48)\rangle,\langle y,(0.38,0.55)\rangle\}}{\mathfrak{q}_{2}}\right\}, \\
\mathbb{N}^{+}(y) & =\left\{\frac{\{\langle w,(0.66,0.57)\rangle,\langle x,(0.63,0.49)\rangle,\langle z,(0.31,0.82)\rangle\}}{\mathfrak{q}_{1}}, \frac{\{\}}{\mathfrak{q}_{2}}\right\}, \\
\mathbb{N}^{+}(z) & =\left\{\frac{\{\langle w,(0.28,0.78)\rangle\}}{\mathfrak{q}_{1}}, \frac{\{\langle v,(0.59,0.42)\rangle,\langle x,(0.58,0.73)\rangle,\langle y,(0.33,0.69)\rangle\}}{\mathfrak{q}_{2}}\right\}
\end{aligned}
$$

The $\mathrm{PFS}_{f}$ in-neighborhoods of $\mathrm{PFS}_{f}$ vertices of $\overrightarrow{\mathbb{G}}$ are

$$
\begin{aligned}
\mathbb{N}^{-}(v) & =\left\{\frac{\{\}}{\mathfrak{q}_{1}}, \frac{\langle\langle w,(0.61,0.49)\rangle,\langle x,(0.63,0.48)\rangle,\langle z,(0.59,0.42)\rangle\}}{\mathfrak{q}_{2}}\right\}, \\
\mathbb{N}^{-}(w) & =\left\{\frac{\{\langle v,(0.51,0.62)\rangle,\langle y,(0.66,0.57)\rangle,\langle z,(0.28,0.78)\rangle\}}{\mathfrak{q}_{1}}, \frac{\{\}}{\mathfrak{q}_{2}}\right\}, \\
\mathbb{N}^{-}(x) & =\left\{\frac{\{\langle v,(0.54,0.64)\rangle,\langle y,(0.63,0.49)\rangle\}}{\mathfrak{q}_{1}}, \frac{\{\langle w,(0.72,0.51)\rangle,\langle z,(0.58,0.73)\rangle\}}{\mathfrak{q}_{2}}\right\}, \\
\mathbb{N}^{-}(y) & =\left\{\frac{\{\langle v,(0.55,0.47)\rangle,\}}{\mathfrak{q}_{1}}, \frac{\{\langle x,(0.38,0.55)\rangle,\langle z,(0.33,0.69)\rangle\}}{\mathfrak{q}_{2}}\right\}, \\
\mathbb{N}^{-}(z) & =\left\{\frac{\{\langle y,(0.31,0.82)\rangle\}}{\mathfrak{q}_{1}}, \frac{\{\langle w,(0.57,0.72)\rangle\}}{\mathfrak{q}_{2}}\right\} .
\end{aligned}
$$

Definition 12 The $\operatorname{PFS}_{f} C G \mathbb{C}(\overrightarrow{\mathbb{G}})=(\mathbb{A}, \mathbb{D}, Q)$ of a $\operatorname{PFS}_{f}$ digraph $\overrightarrow{\mathbb{G}}=(\mathbb{A}, \overrightarrow{\mathbb{B}}, Q)$ is an undirected $\mathrm{PFS}_{f} \mathrm{G}$ that has same $\operatorname{PFS}_{f}$ vertex set as in $\overrightarrow{\mathbb{G}}$ and for all $\mathfrak{q} \in Q$, it has a Pythagorean fuzzy edge between points $x, y \in X$ in $\mathbb{S}(\mathfrak{q})=$ $(\mathbb{A}(\mathfrak{q}), \mathbb{D}(\mathfrak{q}))$ if and only if $\mathbb{N}^{+}(\mathfrak{q})(x) \cap \mathbb{N}^{+}(\mathfrak{q})(y)$ is nonempty set. The membership as well as non-membership grade of edges in $\mathbb{S}(\mathfrak{q})$ are computed as follows:
We can calculate the edge membership and nonmembership values for the corresponding $\operatorname{PFS}_{f} \mathrm{CG} \mathbb{C}(\overrightarrow{\mathbb{G}})=$ $(\mathbb{A}, \mathbb{D}, Q)$ as

$$
\begin{aligned}
& \mathbb{N}^{+}\left(\mathfrak{q}_{1}\right)(v) \cap \mathbb{N}^{+}\left(\mathfrak{q}_{1}\right)(y) \\
& =\{\langle w,(0.51,0.62)\rangle,\langle x,(0.54,0.64)\rangle\} \\
& \Rightarrow \quad h\left(\mathbb{N}^{+}\left(\mathfrak{q}_{1}\right)(v) \cap \mathbb{N}^{+}\left(\mathfrak{q}_{1}\right)(y)\right)=(0.54,0.62)
\end{aligned}
$$



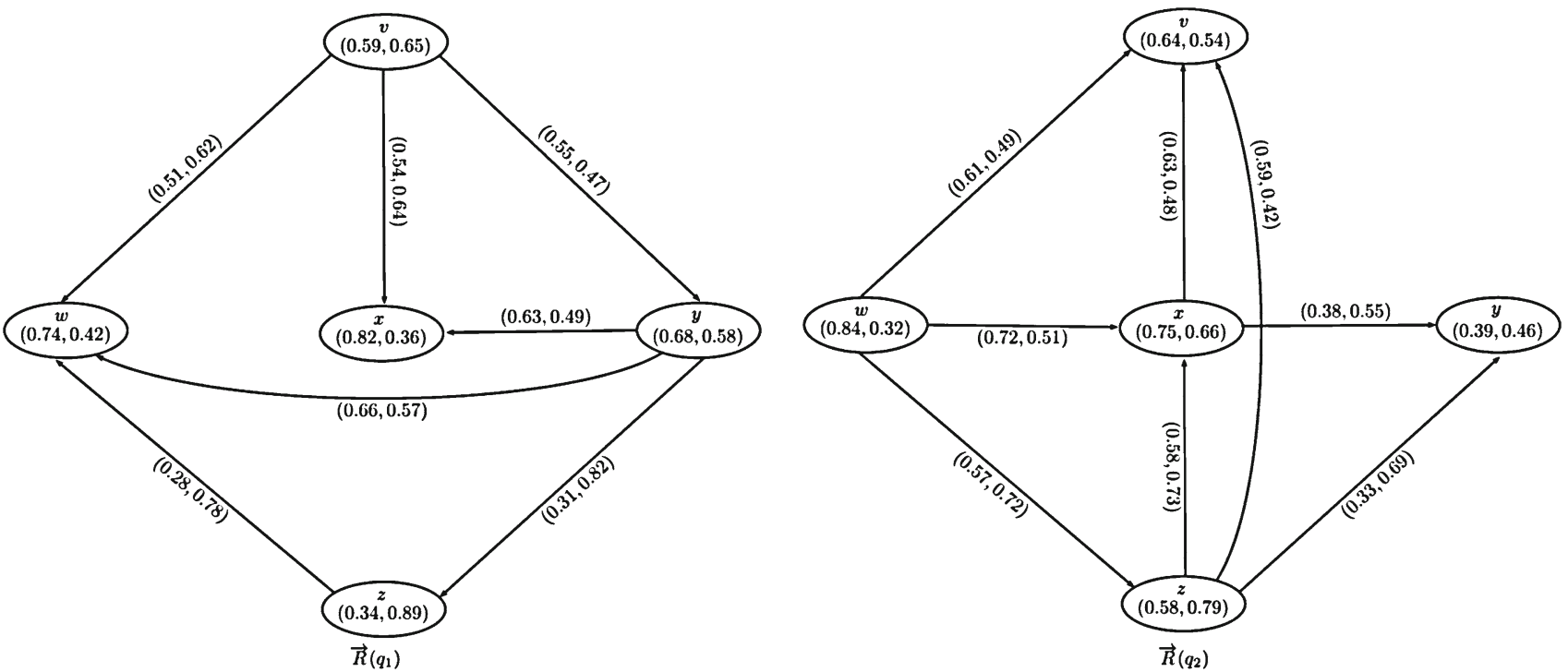

Fig. 2 A PFS $_{f}$ digraph

$$
\left\{\begin{aligned}
\mu_{\mathbb{D}\left(\mathfrak{q}_{1}\right)}(v, y) & =\left(\mu_{\mathbb{A}\left(\mathfrak{q}_{1}\right)}(v) \wedge \mu_{\mathbb{A}\left(\mathfrak{q}_{1}\right)}(y)\right) h_{\mu}\left(\mathbb{N}^{+}\left(\mathfrak{q}_{1}\right)(v)\right. \\
& \left.\times \cap \mathbb{N}^{+}\left(\mathfrak{q}_{1}\right)(y)\right)=(0.59 \wedge 0.68)(0.54) \\
& =0.32, \\
v_{\mathbb{D}\left(\mathfrak{q}_{1}\right)}(v, y) & =\left(v_{\mathbb{A}\left(\mathfrak{q}_{1}\right)}(v) \vee v_{\mathbb{A}\left(\mathfrak{q}_{1}\right)}(y)\right) h_{v}\left(\mathbb{N}^{+}\left(\mathfrak{q}_{1}\right)(v)\right. \\
& \left.\times \cap \mathbb{N}^{+}\left(\mathfrak{q}_{1}\right)(y)\right)=(0.65 \vee 0.58)(0.62) \\
& =0.40,
\end{aligned}\right.
$$

$$
\left\{\begin{aligned}
\mu_{\mathbb{F}(\mathfrak{q})}(x, y) & =\left(\mu_{\mathbb{A}(\mathfrak{q})}(x) \wedge \mu_{\mathbb{A}(\mathfrak{q})}(y)\right) h_{\mu}\left(\mathbb{N}^{-}(\mathfrak{q})(x)\right. \\
& \left.\times \cap \mathbb{N}^{-}(\mathfrak{q})(y)\right), \\
v_{\mathbb{F}(\mathfrak{q})}(x, y) & =\left(v_{\mathbb{A}(\mathfrak{q})}(x) \vee v_{\mathbb{A}(\mathfrak{q})}(y)\right) h_{\nu}\left(\mathbb{N}^{-}(\mathfrak{q})(x)\right. \\
& \left.\times \cap \mathbb{N}^{-}(\mathfrak{q})(y)\right),
\end{aligned}\right.
$$

where $\mu_{\mathbb{D}\left(\mathfrak{q}_{1}\right)}^{2}(v, w)+v_{\mathbb{D}\left(\mathfrak{q}_{1}\right)}^{2}(v, w) \leq 1$. Hence, we have $\mathbb{C}(\overrightarrow{\mathbb{G}})=\left(\mathbb{S}\left(\mathfrak{q}_{1}\right), \mathbb{S}\left(\mathfrak{q}_{2}\right)\right)$ displayed in Fig. 3, where

$$
\begin{aligned}
\mathbb{S}\left(\mathfrak{q}_{1}\right)=( & \left.\mathbb{A}\left(\mathfrak{q}_{1}\right), \mathbb{D}\left(\mathfrak{q}_{1}\right)\right)=(\{\langle v,(0.59,0.65)\rangle, \\
& \langle w,(0.74,0.42)\rangle,\langle x,(0.82,0.36)\rangle, \\
& \langle y,(0.68,0.58)\rangle,\langle z,(0.34,0.89)\rangle\}, \\
\{ & \langle(v, y),(0.32,0.40)\rangle,\langle(v, z),(0.09,0.69)\rangle, \\
& \langle(y, z),(0.09,0.69)\rangle\}), \\
\mathbb{S}\left(\mathfrak{q}_{2}\right)=( & \left.\mathbb{A}\left(\mathfrak{q}_{2}\right), \mathbb{D}\left(\mathfrak{q}_{2}\right)\right)=(\{\langle v,(0.64,0.54)\rangle, \\
& \langle w,(0.84,0.32)\rangle,\langle x,(0.75,0.66)\rangle, \\
& \langle y,(0.39,0.46)\rangle,\langle z,(0.58,0.79)\rangle\}, \\
& \langle(w, z),(0.34,0.39)\rangle,\langle(x, z),(0.19,0.54)\rangle\}) .
\end{aligned}
$$

Definition 13 The $\mathrm{PFS}_{f} E C G \mathbb{E}(\overrightarrow{\mathbb{G}})=(\mathbb{A}, \mathbb{F}, Q)$ of a PFS $\operatorname{digraph} \overrightarrow{\mathbb{G}}=(\mathbb{A}, \overrightarrow{\mathbb{B}}, Q)$ is an undirected $\mathrm{PFS}_{f} \mathrm{G}$ that has same $\operatorname{PFS}_{f}$ vertex set as in $\overrightarrow{\mathbb{G}}$ and for all $\mathfrak{q} \in Q$, it has a Pythagorean fuzzy edge between points $x, y \in X$ in $\mathbb{T}(\mathfrak{q})=(\mathbb{A}(\mathfrak{q}), \mathbb{F}(\mathfrak{q}))$ if and only if $\mathbb{N}^{-}(\mathfrak{q})(x) \cap \mathbb{N}^{-}(\mathfrak{q})(y)$ is non-empty set. The membership as well as non-membership grade of edges in $\mathbb{T}(\mathfrak{q})$ are computed as follows:

such that $0 \leq \mu_{\mathbb{F}(\mathfrak{q})}^{2}(x, y)+v_{\mathbb{F}(\mathfrak{q})}^{2}(x, y) \leq 1$, for all $x, y \in X$ and $\mathfrak{q} \in Q$.

Example 4 The $\mathrm{PFS}_{f}$ ECG of $\vec{G}=(\mathbb{A}, \vec{B}, Q)$ given in Example 2 is $\mathbb{E}(\overrightarrow{\mathbb{G}})=(\mathbb{A}, \mathbb{F}, Q)$ shown in Fig. 4 .

The edge membership and non-membership values of $\mathbb{E}(\overrightarrow{\mathbb{G}})$ are calculated as

$$
\begin{aligned}
& \mathbb{N}^{-}\left(\mathfrak{q}_{1}\right)(w) \cap \mathbb{N}^{-}\left(\mathfrak{q}_{1}\right)(x) \\
& =\{\langle v,(0.51,0.64)\rangle,\langle y,(0.63,0.57)\rangle\} \\
& \Rightarrow \quad h\left(\mathbb{N}^{-}\left(\mathfrak{q}_{1}\right)(w) \cap \mathbb{N}^{-}\left(\mathfrak{q}_{1}\right)(x)\right)=(0.63,0.57) \\
& \left\{\begin{aligned}
\mu_{\mathbb{F}\left(\mathfrak{q}_{1}\right)}(w, x) & =\left(\mu_{\mathbb{A}\left(\mathfrak{q}_{1}\right)}(w) \wedge \mu_{\mathbb{A}\left(\mathfrak{q}_{1}\right)}(x)\right) h_{\mu}\left(\mathbb{N}^{-}\left(\mathfrak{q}_{1}\right)(w)\right. \\
& \left.\times \cap \mathbb{N}^{-}\left(\mathfrak{q}_{1}\right)(x)\right)=(0.74 \wedge 0.82)(0.63) \\
& =0.47, \\
v_{\mathbb{F}\left(\mathfrak{q}_{1}\right)}(w, x) & =\left(v_{\mathbb{A}\left(\mathfrak{q}_{1}\right)}(w) \vee v_{\mathbb{A}\left(\mathfrak{q}_{1}\right)}(x)\right) h_{v}\left(\mathbb{N}^{-}\left(\mathfrak{q}_{1}\right)(w)\right. \\
& \left.\times \cap \mathbb{N}^{-}\left(\mathfrak{q}_{1}\right)(x)\right)=(0.42 \vee 0.36)(0.57) \\
& =0.24,
\end{aligned}\right.
\end{aligned}
$$

where $\mu_{\mathbb{F}\left(\mathfrak{q}_{1}\right)}^{2}(w, x)+v_{\mathbb{F}\left(\mathfrak{q}_{1}\right)}^{2}(w, x) \leq 1$. Thus, we have $\mathbb{E}(\overrightarrow{\mathbb{G}})=\left(\mathbb{T}\left(\mathfrak{q}_{1}\right), \mathbb{T}\left(\mathfrak{q}_{2}\right)\right)$, where

$\mathbb{T}\left(\mathfrak{q}_{1}\right)=\left(\mathbb{A}\left(\mathfrak{q}_{1}\right), \mathbb{F}\left(\mathfrak{q}_{1}\right)\right)=(\{\langle v,(0.59,0.65)\rangle$, 

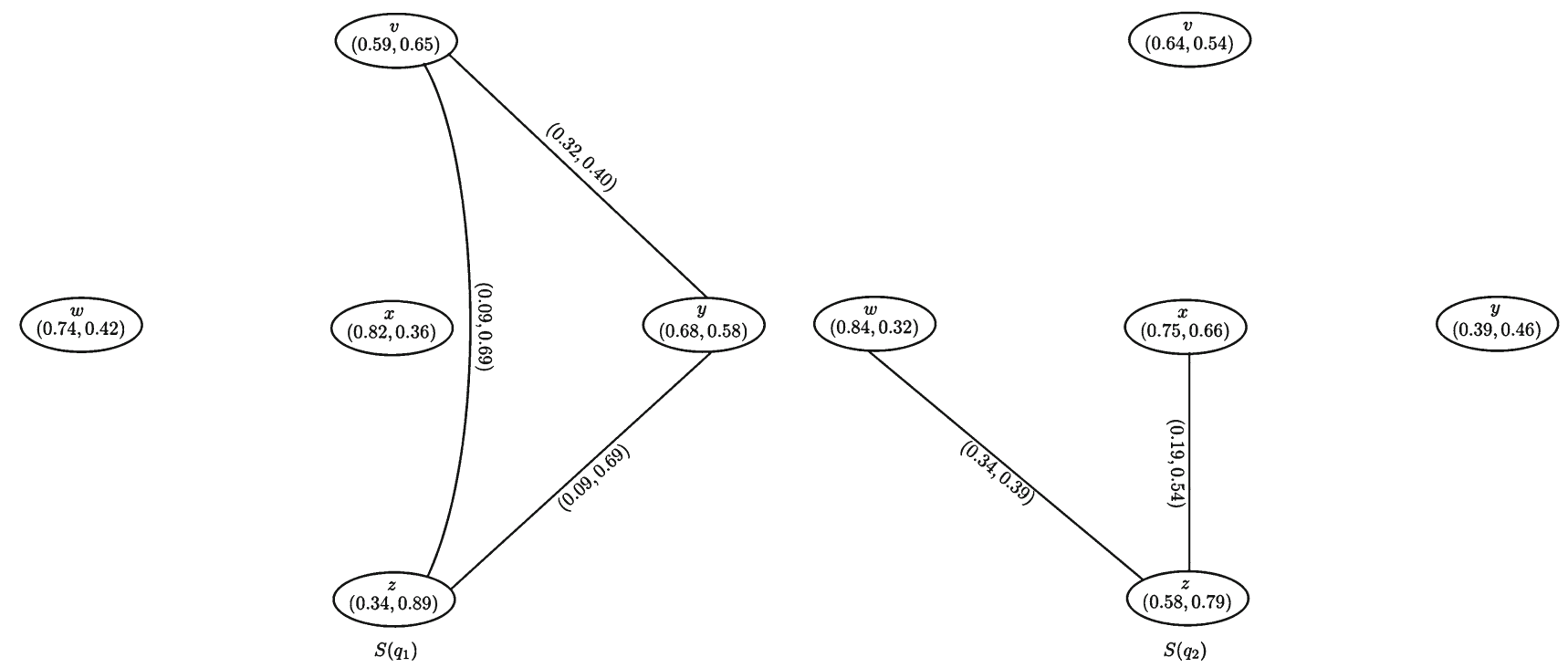

Fig. $3 \mathrm{APFS}_{f} \mathrm{CG}$
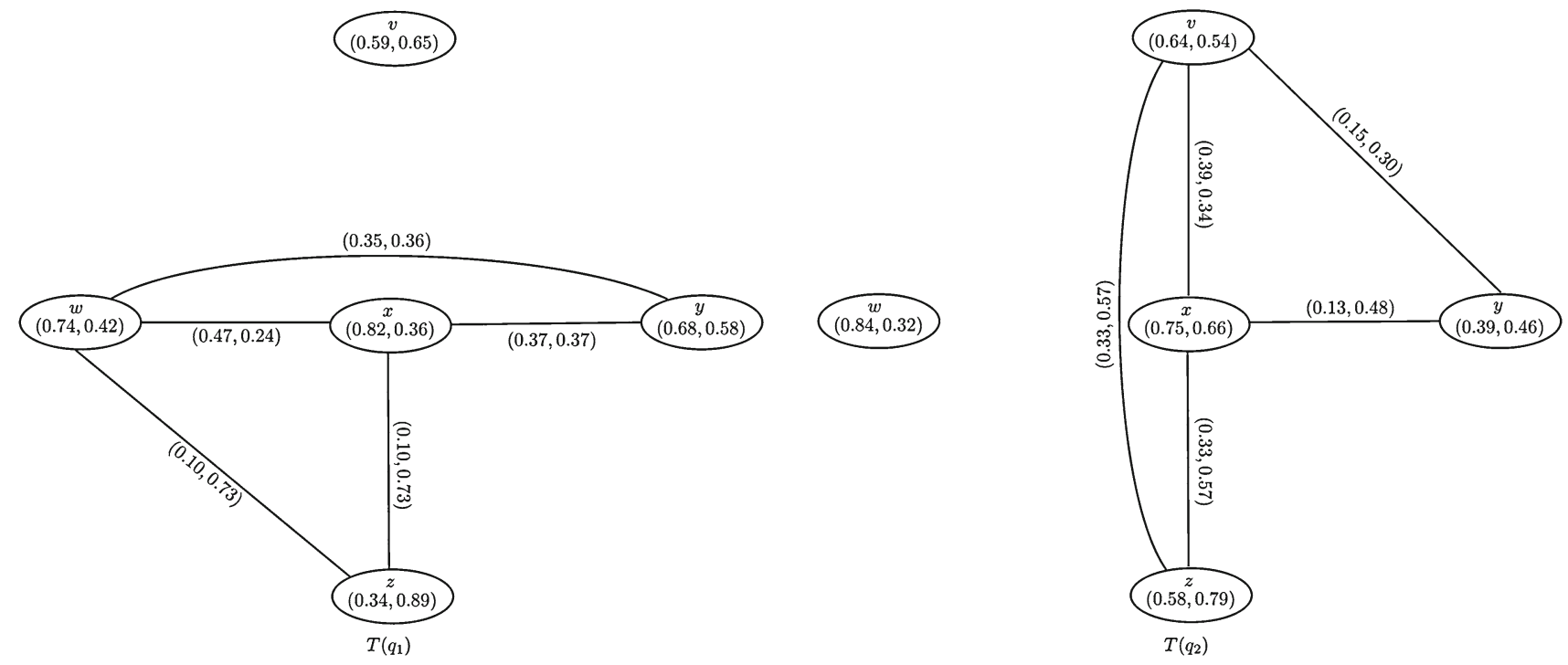

Fig. 4 A PFS ${ }_{f}$ ECG

$$
\begin{aligned}
& \langle w,(0.74,0.42)\rangle,\langle x,(0.82,0.36)\rangle, \\
& \langle y,(0.68,0.58)\rangle,\langle z,(0.34,0.89)\rangle\}, \\
\{ & \langle(w, x),(0.47,0.24)\rangle,\langle(w, y),(0.35,0.36)\rangle, \\
& \langle(w, z),(0.10,0.73)\rangle,\langle(x, y),(0.37,0.37)\rangle, \\
& \langle(x, z),(0.10,0.73)\rangle\}), \\
\mathbb{T}\left(\mathfrak{q}_{2}\right)= & \left(\mathbb{A}\left(\mathfrak{q}_{2}\right), \mathbb{F}\left(\mathfrak{q}_{2}\right)\right)=(\{\langle v,(0.64,0.54)\rangle, \\
& \langle w,(0.84,0.32)\rangle,\langle x,(0.75,0.66)\rangle, \\
& \langle y,(0.39,0.46)\rangle,\langle z,(0.58,0.79)\rangle\}, \\
\{ & \langle(v, x),(0.39,0.34)\rangle,\langle(v, y),(0.15,0.30)\rangle, \\
& \langle(v, z),(0.33,0.57)\rangle,\langle(x, y),(0.13,0.48)\rangle, \\
& \langle(x, z),(0.33,0.57)\rangle\}) .
\end{aligned}
$$

Theorem 1 Let $\overrightarrow{\mathbb{G}}=(\mathbb{A}, \overrightarrow{\mathbb{B}}, Q)$ be a PFS digraph on $a$ non-void set $X$.

1. If $\operatorname{supp}\left(\mathbb{N}^{+}(\mathfrak{q})(x) \cap \mathbb{N}^{+}(\mathfrak{q})(y)\right)$ is a singleton subset of $X$, then the Pythagorean fuzzy edge $(x, y)$ of $\mathbb{S}(\mathfrak{q})=$ $(\mathbb{A}(\mathfrak{q}), \mathbb{D}(\mathfrak{q}))$ in $\mathbb{C}(\overrightarrow{\mathbb{G}})$ is independent strong if and only if $\left|\mathbb{N}^{+}(\mathfrak{q})(x) \cap \mathbb{N}^{+}(\mathfrak{q})(y)\right|_{\mu}>0.5$ and $\mid \mathbb{N}^{+}(\mathfrak{q})(x) \cap$ $\left.\mathbb{N}^{+}(\mathfrak{q})(y)\right|_{v}<0.5$.

2. If $\operatorname{supp}\left(\mathbb{N}^{-}(\mathfrak{q})(x) \cap \mathbb{N}^{-}(\mathfrak{q})(y)\right)$ is a singleton subset of $X$, then the Pythagorean fuzzy edge $(x, y)$ of $\mathbb{T}(\mathfrak{q})=$ $(\mathbb{A}(\mathfrak{q}), \mathbb{F}(\mathfrak{q}))$ in $\mathbb{E}(\overrightarrow{\mathbb{G}})$ is independent strong if and only 
if $\left|\mathbb{N}^{-}(\mathfrak{q})(x) \cap \mathbb{N}^{-}(\mathfrak{q})(y)\right|_{\mu}>0.5$ and $\mid \mathbb{N}^{-}(\mathfrak{q})(x) \cap$ $\left.\mathbb{N}^{-}(\mathfrak{q})(y)\right|_{v}<0.5$.

In general if, in both cases, the sufficient condition holds relative to all attributes $\mathfrak{q}$, then the $\operatorname{PFS}_{f}$ edge is said to be strong and vice versa.

Proof Let $\overrightarrow{\mathbb{G}}=(\mathbb{A}, \overrightarrow{\mathbb{B}}, Q)$ be a PFS $f$ digraph. Assume that $z$ is the only element with membership degree $\mu_{(\mathfrak{q})}(z)$ and non-membership degree $v_{(\mathfrak{q})}(z)$ such that $\mathbb{N}^{+}(\mathfrak{q})(x) \cap$ $\mathbb{N}^{+}(\mathfrak{q})(y)=\left\{\left\langle z,\left(\mu_{(\mathfrak{q})}(z), v_{(\mathfrak{q})}(z)\right)\right\rangle\right\}$. Clearly $\operatorname{supp}\left(\mathbb{N}^{+}(\mathfrak{q})(x)\right.$ $\left.\cap \mathbb{N}^{+}(\mathfrak{q})(y)\right)=\{z\}$. Observe that $\left|\mathbb{N}^{+}(\mathfrak{q})(x) \cap \mathbb{N}^{+}(\mathfrak{q})(y)\right|_{\mu}=$ $\mu_{(\mathfrak{q})}(z)=h_{\mu}\left(\mathbb{N}^{+}(\mathfrak{q})(x) \cap \mathbb{N}^{+}(\mathfrak{q})(y)\right)$ and $\mid \mathbb{N}^{+}(\mathfrak{q})(x) \cap$ $\left.\mathbb{N}^{+}(\mathfrak{q})(y)\right|_{\nu}=v_{(\mathfrak{q})}(z)=h_{v}\left(\mathbb{N}^{+}(\mathfrak{q})(x) \cap \mathbb{N}^{+}(\mathfrak{q})(y)\right)$.

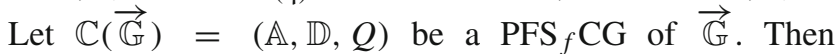
$\mu_{\mathbb{D}(\mathfrak{q})}(x, y)=\left(\mu_{\mathbb{A}(\mathfrak{q})}(x) \wedge \mu_{\mathbb{A}(\mathfrak{q})}(y)\right) \times \mu_{(\mathfrak{q})}(z)$ and $v_{\mathbb{D}(\mathfrak{q})}$ $(x, y)=\left(v_{\mathbb{A}(\mathfrak{q})}(x) \vee v_{\mathbb{A}(\mathfrak{q})}(y)\right) \times v_{(\mathfrak{q})}(z)$ give the membership and non-membership values of edge $(x, y)$ in $\mathbb{S}(\mathfrak{q})=$ $(\mathbb{A}(\mathfrak{q}), \mathbb{D}(\mathfrak{q}))$. As a consequence, we have $\frac{\mu_{\mathbb{D}(\mathfrak{q})}(x, y)}{\mu_{\mathbb{A}(\mathfrak{q})}(x) \wedge \mu_{\mathbb{A}(\mathfrak{q})}(y)}=$ $\mu_{(\mathfrak{q})}(z)$ and $\frac{v_{\mathbb{D}(\mathfrak{q})}(x, y)}{v_{\mathbb{A}(\mathfrak{q})}(x) \vee v_{\mathbb{A}(\mathfrak{q})}(y)}=v_{(\mathfrak{q})}(z)$.

Assume the Pythagorean fuzzy edge $(x, y)$ of $\mathbb{S}(\mathfrak{q})$ in $\mathbb{C}(\overrightarrow{\mathbb{G}})$ to be independent strong so that $\mu_{\mathbb{D}(\mathfrak{q})}(x, y)>$ $\frac{1}{2}\left(\mu_{\mathbb{A}(\mathfrak{q})}(x) \wedge \mu_{\mathbb{A}(\mathfrak{q})}(y)\right)$ and $v_{\mathbb{D}(\mathfrak{q})}(x, y)<\frac{1}{2}\left(v_{\mathbb{A}(\mathfrak{q})}(x) \vee\right.$ $\left.v_{\mathbb{A}(\mathfrak{q})}(y)\right)$, i.e., $\frac{\mu_{\mathbb{D}(\mathfrak{q})}(x, y)}{\mu_{\mathbb{A}(\mathfrak{q})}(x) \wedge \mu_{\mathbb{A}(\mathfrak{q})}(y)}>\frac{1}{2}$ and $\frac{v_{\mathbb{D}(\mathfrak{q})}(x, y)}{v_{\mathbb{A}(\mathfrak{q})}(x) \vee v_{\mathbb{A}(\mathfrak{q})}(y)}<$ $\frac{1}{2}$. Both results combine to give $\mu_{(\mathfrak{q})}(z)>\frac{1}{2}$ as well as $v_{(\mathfrak{q})}(z)<\frac{1}{2}$. Consequently, $\left|\mathbb{N}^{+}(\mathfrak{q})(x) \cap \mathbb{N}^{+}(\mathfrak{q})(y)\right|_{\mu}>\frac{1}{2}$ and $\left|\mathbb{N}^{+}(\mathfrak{q})(x) \cap \mathbb{N}^{+}(\mathfrak{q})(y)\right|_{v}<\frac{1}{2}$.

Conversely, let $\left|\mathbb{N}^{+}(\mathfrak{q})(x) \cap \mathbb{N}^{+}(\mathfrak{q})(y)\right|_{\mu}>\frac{1}{2}$ and $\left|\mathbb{N}^{+}(\mathfrak{q})(x) \cap \mathbb{N}^{+}(\mathfrak{q})(y)\right|_{\nu}<\frac{1}{2}$ which implies $\mu_{(\mathfrak{q})}(z)>\frac{1}{2}$ and $v_{(\mathfrak{q})}(z)<\frac{1}{2}$, respectively. Both these relations combine to give $\frac{\mu_{\mathbb{D}(\mathfrak{q})}(x, y)}{\mu_{\mathbb{A}(\mathfrak{q})}(x) \wedge \mu_{\mathbb{A}(\mathfrak{q})}(y)}>\frac{1}{2}$ and $\frac{v_{\mathbb{D}(\mathfrak{q})}(x, y)}{v_{\mathbb{A}(\mathfrak{q})}(x) \vee v_{\mathbb{A}(\mathfrak{q})}(y)}<\frac{1}{2}$. It follows that the edge $(x, y)$ of $\mathbb{S}(\mathfrak{q})=(\mathbb{A}(\mathfrak{q}), \mathbb{D}(\mathfrak{q}))$ in $\mathbb{C}(\overrightarrow{\mathbb{G}})$ is independent strong.

The strategy of the proof of (2) is similar to the above.

Theorem 2 Let $\overrightarrow{\mathbb{G}}=(\mathbb{A}, \overrightarrow{\mathbb{B}}, Q)$ be a PFS digraph on $X$.

1. If all PFS $f$ directed edges of $\overrightarrow{\mathbb{G}}$ are independent strong, then $\min _{\mathfrak{q}}\left(\frac{\mu_{\mathbb{D}(\mathfrak{q})}(x, y)}{\left[\mu_{\mathbb{A}(\mathfrak{q})}(x) \wedge \mu_{\mathbb{A}(\mathfrak{q})}(y)\right]^{2}}\right)>\frac{1}{2}$ as well as $\max _{\mathfrak{q}}\left(\frac{v_{\mathbb{D}(\mathfrak{q})}(x, y)}{\left[v_{\mathbb{A}(\mathfrak{q})}(x) \vee v_{\mathbb{A}(\mathfrak{q})}(y)\right]^{2}}\right)<\frac{1}{2}$ for all PFS $f$ edges $(x, y)$ in $\mathbb{C}(\overrightarrow{\mathbb{G}})$.

2. If all PFS directed edges of $\overrightarrow{\mathbb{G}}$ are independent strong, then $\min _{\mathfrak{q}}\left(\frac{\mu_{\mathbb{F}(\mathfrak{q})}(x, y)}{\left[\mu_{\mathbb{A}(\mathfrak{q})}(x) \wedge \mu_{\mathbb{A}(\mathfrak{q})}(y)\right]^{2}}\right)>\frac{1}{2}$ as well as $\max _{\mathfrak{q}}\left(\frac{v_{\mathbb{F}(\mathfrak{q})}(x, y)}{\left[v_{\mathbb{A}(\mathfrak{q})}(x) \vee v_{\mathbb{A}(\mathfrak{q})}(y)\right]^{2}}\right)<\frac{1}{2}$ for all PFS $f$ edges $(x, y)$ in $\mathbb{E}(\overrightarrow{\mathbb{G}})$.
Theorem 3 The PFS ${ }_{f} C G$ and $P F S_{f} E C G$ are equal for a complete $\mathrm{PFS}_{f}$ digraph.

Proof Let us consider a complete $\mathrm{PFS}_{f}$ digraph $\overrightarrow{\mathbb{G}}=$ $(\mathbb{A}, \overrightarrow{\mathbb{B}}, Q)$ on $X$ and assume that $\mathbb{C}(\overrightarrow{\mathbb{G}})=(\mathbb{A}, \mathbb{D}, Q)$ and $\mathbb{E}(\overrightarrow{\mathbb{G}})=(\mathbb{A}, \mathbb{F}, Q)$ are the corresponding $\operatorname{PFS}_{f} \mathrm{CG}$ as well as $\mathrm{PFS}_{f} \mathrm{ECG}$, respectively. Since in a complete $\mathrm{PFS}_{f} \mathrm{G}$, $\mathbb{N}_{m}^{+}(x)=\mathbb{N}_{m}^{-}(x)$ which certainly means that $\mathbb{N}_{m}^{+}(\mathfrak{q})(x)=$ $\mathbb{N}_{m}^{-}(\mathfrak{q})(x)$ as well as $\mathbb{N}_{m}^{+}(\mathfrak{q})(x) \cap \mathbb{N}_{m}^{+}(\mathfrak{q})(y)=\mathbb{N}_{m}^{-}(\mathfrak{q})(x) \cap$ $\mathbb{N}_{m}^{-}(\mathfrak{q})(y)$ also holds for all $x, y \in X, \mathfrak{q} \in Q$. Consequently,

$\left\{\begin{array}{l}\left(\mu_{\mathbb{A}(\mathfrak{q})}(x) \wedge \mu_{\mathbb{A}(\mathfrak{q})}(y)\right) h_{\mu}\left(\mathbb{N}^{+}(\mathfrak{q})(x) \cap \mathbb{N}^{+}(\mathfrak{q})(y)\right) \\ =\left(\mu_{\mathbb{A}(\mathfrak{q})}(x) \wedge \mu_{\mathbb{A}(\mathfrak{q})}(y)\right) h_{\mu}\left(\mathbb{N}^{-}(\mathfrak{q})(x) \cap \mathbb{N}^{-}(\mathfrak{q})(y)\right), \\ \left(v_{\mathbb{A}(\mathfrak{q})}(x) \vee v_{\mathbb{A}(\mathfrak{q})}(y)\right) h_{v}\left(\mathbb{N}^{+}(\mathfrak{q})(x) \cap \mathbb{N}^{+}(\mathfrak{q})(y)\right) \\ =\left(v_{\mathbb{A}(\mathfrak{q})}(x) \vee v_{\mathbb{A}(\mathfrak{q})}(y)\right) h_{v}\left(\mathbb{N}^{-}(\mathfrak{q})(x) \cap \mathbb{N}^{-}(\mathfrak{q})(y)\right) .\end{array}\right.$

This implies $\mu_{\mathbb{D}(\mathfrak{q})}(x, y)=\mu_{\mathbb{F}(\mathfrak{q})}(x, y)$ and $v_{\mathbb{D}(\mathfrak{q})}(x, y)=$ $v_{\mathbb{F}(\mathfrak{q})}(x, y)$, where $\mu_{\mathbb{D}(\mathfrak{q})}^{2}(x, y)+v_{\mathbb{D}(\mathfrak{q})}^{2}(x, y) \leq 1$ for all $x, y \in X$ and $\mathfrak{q} \in Q$. Thus, $\mathbb{C}(\overrightarrow{\mathbb{G}})=\mathbb{E}(\overrightarrow{\mathbb{G}})$.

Definition 14 For a non-negative real number $k$, the Pythagorean fuzzy soft $k$-competition graph $\left(\mathrm{PFS}_{f} k-C G\right)$

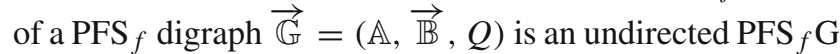
$\mathbb{C}_{k}(\overrightarrow{\mathbb{G}})=(\mathbb{A}, \mathbb{D}, Q)$ which has the same $\operatorname{PFS}_{f}$ vertex set as in $\overrightarrow{\mathbb{G}}$ and for all $\mathfrak{q} \in Q$, it has a Pythagorean fuzzy edge between points $x, y \in X$ in $\mathbb{S}(\mathfrak{q})=(\mathbb{A}(\mathfrak{q}), \mathbb{D}(\mathfrak{q}))$ if and only if $\left|\mathbb{N}^{+}(\mathfrak{q})(x) \cap \mathbb{N}^{+}(\mathfrak{q})(y)\right|_{\mu}>k$ and $\mid \mathbb{N}^{+}(\mathfrak{q})(x) \cap$ $\left.\mathbb{N}^{+}(\mathfrak{q})(y)\right|_{v}>k$. The membership as well as non-membership grade of edges in $\mathbb{S}(\mathfrak{q})$ are computed as follows:

$$
\left\{\begin{aligned}
\mu_{\mathbb{D}(\mathfrak{q})}(x, y) & =\frac{k_{1}-k}{k_{1}}\left(\mu_{\mathbb{A}(\mathfrak{q})}(x) \wedge \mu_{\mathbb{A}(\mathfrak{q})}(y)\right) h_{\mu}\left(\mathbb{N}^{+}(\mathfrak{q})(x)\right. \\
& \left.\cap \mathbb{N}^{+}(\mathfrak{q})(y)\right), \\
v_{\mathbb{D}(\mathfrak{q})}(x, y) & =\frac{k_{2}-k}{k_{2}}\left(v_{\mathbb{A}(\mathfrak{q})}(x) \vee v_{\mathbb{A}(\mathfrak{q})}(y)\right) h_{v}\left(\mathbb{N}^{+}(\mathfrak{q})(x)\right. \\
& \left.\cap \mathbb{N}^{+}(\mathfrak{q})(y)\right),
\end{aligned}\right.
$$

such that $0 \leq \mu_{\mathbb{D}(\mathfrak{q})}^{2}(x, y)+v_{\mathbb{D}(\mathfrak{q})}^{2}(x, y) \leq 1$ for all $x, y \in$ $X, \mathfrak{q} \in Q$, where $k_{1}=\left|\mathbb{N}^{+}(\mathfrak{q})(x) \cap \mathbb{N}^{+}(\mathfrak{q})(y)\right|_{\mu}, k_{2}=$ $\left|\mathbb{N}^{+}(\mathfrak{q})(x) \cap \mathbb{N}^{+}(\mathfrak{q})(y)\right|_{\nu}$.

Example 5 Consider the $\operatorname{PFS}_{f}$ digraph $\overrightarrow{\mathbb{G}}=(\mathbb{A}, \overrightarrow{\mathbb{B}}, Q)$ of Example 2.

We can construct the corresponding $\operatorname{PFS}_{f} 0.3-\mathrm{CG} \mathbb{C}_{0.3}(\overrightarrow{\mathbb{G}})$ $=(\mathbb{A}, \mathbb{D}, Q)$, whose edge membership and non-membership degrees are computed as follows:

$k_{1}=\left|\mathbb{N}^{+}\left(\mathfrak{q}_{1}\right)(v) \cap \mathbb{N}^{+}\left(\mathfrak{q}_{1}\right)(y)\right|_{\mu}=1.05$,

$k_{2}=\left|\mathbb{N}^{+}\left(\mathfrak{q}_{1}\right)(v) \cap \mathbb{N}^{+}\left(\mathfrak{q}_{1}\right)(y)\right|_{v}=1.26$, 


$$
\left\{\begin{array}{l}
\mu_{\mathbb{D}\left(\mathfrak{q}_{1}\right)}(v, y) \\
=\frac{k_{1}-k}{k_{1}}\left(\mu_{\mathbb{A}\left(\mathfrak{q}_{1}\right)}(v) \wedge \mu_{\mathbb{A}\left(\mathfrak{q}_{1}\right)}(y)\right) h_{\mu}\left(\mathbb{N}^{+}\left(\mathfrak{q}_{1}\right)(v)\right. \\
\left.\cap \mathbb{N}^{+}\left(\mathfrak{q}_{1}\right)(y)\right)=(0.71)(0.59 \wedge 0.68)(0.54)=0.23, \\
v_{\mathbb{D}\left(\mathfrak{q}_{1}\right)}(v, y) \\
=\frac{k_{2}-k}{k_{2}}\left(v_{\mathbb{A}\left(\mathfrak{q}_{1}\right)}(v) \vee v_{\mathbb{A}\left(\mathfrak{q}_{1}\right)}(y)\right) h_{v}\left(\mathbb{N}^{+}\left(\mathfrak{q}_{1}\right)(v)\right. \\
\left.\cap \mathbb{N}^{+}\left(\mathfrak{q}_{1}\right)(y)\right)=(0.76)(0.65 \vee 0.58)(0.62)=0.31,
\end{array}\right.
$$

where $\mu_{\mathbb{D}\left(\mathfrak{q}_{1}\right)}^{2}(v, w)+v_{\mathbb{D}\left(\mathfrak{q}_{1}\right)}^{2}(v, w) \leq 1$. The $\mathrm{PFS}_{f} \mathrm{G}$ $C_{0.3}(\overrightarrow{\mathbb{G}})=(\mathbb{A}, \mathbb{D}, Q)$ is given in Fig. 5 .

Definition 15 For a non-negative real number $k$, the Pythagorean fuzzy soft k-economic competition graph $\left(\mathrm{PFS}_{f}\right.$ $k$-economic $C G)$ of a $\mathrm{PFS}_{f}$ digraph $\overrightarrow{\mathbb{G}}=(\mathbb{A}, \overrightarrow{\mathbb{B}}, Q)$ is an undirected $\operatorname{PFS}_{f} \mathrm{G} \mathbb{E}_{k}(\overrightarrow{\mathbb{G}})=(\mathbb{A}, \mathbb{F}, Q)$ which has the same $\mathrm{PFS}_{f}$ vertex set as in $\overrightarrow{\mathbb{G}}$ and for all $\mathfrak{q} \in Q$, the Pythagorean fuzzy points $x, y \in X$ are joined in $\mathbb{T}(\mathfrak{q})=$ $(\mathbb{A}(\mathfrak{q}), \mathbb{F}(\mathfrak{q}))$ if and only if $\left|\mathbb{N}^{-}(\mathfrak{q})(x) \cap \mathbb{N}^{-}(\mathfrak{q})(y)\right|_{\mu}>k$ and $\left|\mathbb{N}^{-}(\mathfrak{q})(x) \cap \mathbb{N}^{-}(\mathfrak{q})(y)\right|_{\mathcal{V}}>k$. The membership as well as non-membership grade of edges in $\mathbb{T}(\mathfrak{q})$ are computed as follows:

$$
\left\{\begin{aligned}
\mu_{\mathbb{F}(\mathfrak{q})}(x, y) & =\frac{k_{1}-k}{k_{1}}\left(\mu_{\mathbb{A}(\mathfrak{q})}(x) \wedge \mu_{\mathbb{A}(\mathfrak{q})}(y)\right) h_{\mu}\left(\mathbb{N}^{-}(\mathfrak{q})(x)\right. \\
& \left.\cap \mathbb{N}^{-}(\mathfrak{q})(y)\right), \\
v_{\mathbb{F}(\mathfrak{q})}(x, y) & =\frac{k_{2}-k}{k_{2}}\left(\nu_{\mathbb{A}(\mathfrak{q})}(x) \vee v_{\mathbb{A}(\mathfrak{q})}(y)\right) h_{v}\left(\mathbb{N}^{-}(\mathfrak{q})(x)\right. \\
& \left.\cap \mathbb{N}^{-}(\mathfrak{q})(y)\right),
\end{aligned}\right.
$$

such that $0 \leq \mu_{\mathbb{F}(\mathfrak{q})}^{2}(x, y)+v_{\mathbb{F}(\mathfrak{q})}^{2}(x, y) \leq 1$ for all $x, y \in$ $X, \mathfrak{q} \in Q$, where $k_{1}=\left|\mathbb{N}^{-}(\mathfrak{q})(x) \cap \mathbb{N}^{-}(\mathfrak{q})(y)\right|_{\mu}, k_{2}=$ $\left|\mathbb{N}^{-}(\mathfrak{q})(x) \cap \mathbb{N}^{-}(\mathfrak{q})(y)\right|_{\nu}$.

Example 6 Consider the $\mathrm{PFS}_{f}$ digraph $\overrightarrow{\mathbb{G}}=(\mathbb{A}, \overrightarrow{\mathbb{B}}, Q)$ of Example 2.

We can construct the corresponding $\mathrm{PFS}_{f} 0.5$-economic CG $\mathbb{E}_{0.5}(\overrightarrow{\mathbb{G}})=(\mathbb{A}, \mathbb{F}, Q)$, whose edge membership and non-membership degrees are computed as

$$
\begin{aligned}
& k_{1}=\left|\mathbb{N}^{-}\left(\mathfrak{q}_{1}\right)(w) \cap \mathbb{N}^{-}\left(\mathfrak{q}_{1}\right)(x)\right|_{\mu}=1.14, \\
& k_{2}=\left|\mathbb{N}^{-}\left(\mathfrak{q}_{1}\right)(w) \cap \mathbb{N}^{-}\left(\mathfrak{q}_{1}\right)(x)\right|_{\nu}=1.21, \\
& \left\{\begin{array}{l}
\mu \mathbb{F}\left(\mathfrak{q}_{1}\right)(w, x) \\
=\frac{k_{1}-k}{k_{1}}\left(\mu_{\mathbb{A}\left(\mathfrak{q}_{1}\right)}(w) \wedge \mu_{\mathbb{A}\left(\mathfrak{q}_{1}\right)}(x)\right) h_{\mu}\left(\mathbb{N}^{-}\left(\mathfrak{q}_{1}\right)(w)\right. \\
\left.\cap \mathbb{N}^{-}\left(\mathfrak{q}_{1}\right)(x)\right)=(0.56)(0.74 \wedge 0.82)(0.63)=0.26, \\
v_{\mathbb{F}\left(\mathfrak{q}_{1}\right)}(w, x) \\
=\frac{k_{2}-k}{k_{2}}\left(v_{\mathbb{A}\left(\mathfrak{q}_{1}\right)}(w) \vee v_{\mathbb{A}\left(\mathfrak{q}_{1}\right)}(x)\right) h_{\nu}\left(\mathbb{N}^{-}\left(\mathfrak{q}_{1}\right)(w)\right. \\
\left.\cap \mathbb{N}^{-}\left(\mathfrak{q}_{1}\right)(x)\right)=(0.59)(0.42 \vee 0.36)(0.57)=0.14,
\end{array}\right.
\end{aligned}
$$

where $\mu_{\mathbb{F}\left(\mathfrak{q}_{1}\right)}^{2}(w, x)+v_{\mathbb{F}\left(\mathfrak{q}_{1}\right)}^{2}(w, x) \leq 1$. The $\mathrm{PFS}_{f} \mathrm{G}$ $E_{0.5}(\overrightarrow{\mathbb{G}})=(\mathbb{A}, \mathbb{F}, Q)$ is given in Fig. 6 .
Theorem 4 Let $\overrightarrow{\mathbb{G}}=(\mathbb{A}, \overrightarrow{\mathbb{B}}, Q)$ be a PFS $f$ digraph on $X$.

1. If $h_{\mu}\left(\mathbb{N}^{+}(\mathfrak{q})(x) \cap \mathbb{N}^{+}(\mathfrak{q})(y)\right)=1=h_{\nu}\left(\mathbb{N}^{+}(\mathfrak{q})(x)\right.$ $\left.\cap \mathbb{N}^{+}(\mathfrak{q})(y)\right), \quad\left|\mathbb{N}^{+}(\mathfrak{q})(x) \cap \mathbb{N}^{+}(\mathfrak{q})(y)\right|_{\mu}>2 k$ and $\left|\mathbb{N}^{+}(\mathfrak{q})(x) \cap \mathbb{N}^{+}(\mathfrak{q})(y)\right|_{v}<2 k$, then the Pythagorean fuzzy edge $(x, y)$ of $\mathbb{S}(\mathfrak{q})=(\mathbb{A}(\mathfrak{q}), \mathbb{D}(\mathfrak{q}))$ in $\mathbb{C}_{k}(\overrightarrow{\mathbb{G}})$ is independent strong.

2. If $h_{\mu}\left(\mathbb{N}^{-}(\mathfrak{q})(x) \cap \mathbb{N}^{-}(\mathfrak{q})(y)\right)=1=h_{v}\left(\mathbb{N}^{-}(\mathfrak{q})(x)\right.$ $\left.\cap \mathbb{N}^{-}(\mathfrak{q})(y)\right), \quad\left|\mathbb{N}^{-}(\mathfrak{q})(x) \cap \mathbb{N}^{-}(\mathfrak{q})(y)\right|_{\mu}>2 k$ and $\left|\mathbb{N}^{-}(\mathfrak{q})(x) \cap \mathbb{N}^{-}(\mathfrak{q})(y)\right|_{v}<2 k$, then the Pythagorean fuzzy edge $(x, y)$ of $\mathbb{T}(\mathfrak{q})=(\mathbb{A}(\mathfrak{q}), \mathbb{F}(\mathfrak{q}))$ in $\mathbb{E}_{k}(\overrightarrow{\mathbb{G}})$ is independent strong.

In general if, in both cases, the statement holds for all parameters $\mathfrak{q}$, then $(x, y)$ is said to be independent strong PFS edge.

Proof Let $\overrightarrow{\mathbb{G}}=(\mathbb{A}, \overrightarrow{\mathbb{B}}, Q)$ be a $\mathrm{PFS}_{f}$ digraph and let $\mathbb{E}_{k}(\overrightarrow{\mathbb{G}})=(\mathbb{A}, \mathbb{F}, Q)$ be the corresponding $\mathrm{PFS}_{f} k$-economic CG. Given that for $x, y \in X$ and $\mathfrak{q} \in Q, h_{\mu}\left(\mathbb{N}^{-}(\mathfrak{q})(x) \cap\right.$ $\left.\mathbb{N}^{-}(\mathfrak{q})(y)\right)=1$ and $k_{1}=\left|\mathbb{N}^{-}(\mathfrak{q})(x) \cap \mathbb{N}^{-}(\mathfrak{q})(y)\right|_{\mu}>$ $2 k$. Combining both these relations to the definition of $\mu_{\mathbb{F}(\mathfrak{q})}(x, y)$, we have $\mu_{\mathbb{F}(\mathfrak{q})}(x, y)=\frac{k_{1}-k}{k_{1}}\left(\mu_{\mathbb{A}(\mathfrak{q})}(x) \wedge\right.$ $\left.\mu_{\mathbb{A}(\mathfrak{q})}(y)\right)$. This yields $\frac{\mu_{\mathbb{F}(\mathfrak{q})}(x, y)}{\mu_{\mathbb{A}(\mathfrak{q})}(x) \wedge \mu_{\mathbb{A}(\mathfrak{q})}(y)}=\frac{k_{1}-k}{k_{1}}>\frac{k_{1}-\frac{k_{1}}{2}}{k_{1}}=$ $\frac{1}{2}$. Now let $h_{\nu}\left(\mathbb{N}^{-}(\mathfrak{q})(x) \cap \mathbb{N}^{-}(\mathfrak{q})(y)\right)=1$ and $k_{2}=$ $\left|\mathbb{N}^{-}(\mathfrak{q})(x) \cap \mathbb{N}^{-}(\mathfrak{q})(y)\right|_{\mathcal{v}}<2 k$, for $x, y \in X$ and $\mathfrak{q} \in$ $Q$. The definition of $v_{\mathbb{F}(\mathfrak{q})}(x, y)$ becomes $v_{\mathbb{F}(\mathfrak{q})}(x, y)=$ $\frac{k_{2}-k}{k_{2}}\left(v_{\mathbb{A}(\mathfrak{q})}(x) \vee v_{\mathbb{A}(\mathfrak{q})}(y)\right)$ which implies $\frac{v_{\mathbb{F}(\mathfrak{q})}(x, y)}{v_{\mathbb{A}(\mathfrak{q})}(x) \vee v_{\mathbb{A}(\mathfrak{q})}(y)}=$ $\frac{k_{2}-k}{k_{2}}<\frac{k_{2}-\frac{k_{2}}{2}}{k_{2}}=\frac{1}{2}$ which shows that the Pythagorean fuzzy edge $(x, y)$ is independent strong in the Pythagorean fuzzy subgraph $\mathbb{T}(\mathfrak{q})$ of $\mathbb{E}_{k}(\overrightarrow{\mathbb{G}})$.

The proof to statement (1) is similar.

Definition 16 Let $\overrightarrow{\mathbb{G}}=(\mathbb{A}, \overrightarrow{\mathbb{B}}, Q)$ be a $\mathrm{PFS}_{f}$ digraph on universal set $X$. The $m$-step $\operatorname{PFS}_{f}$ digraph of $\overrightarrow{\mathbb{G}}$ is a $\operatorname{PFS}_{f}$ digraph $\overrightarrow{\mathbb{G}}_{m}=(\mathbb{A}, \overrightarrow{\mathbb{H}}, Q)$ which has the same $\mathrm{PFS}_{f}$ vertex set as in $\overrightarrow{\mathbb{G}}$ and for all $\mathfrak{q} \in Q$, the Pythagorean fuzzy points $x, y \in X$ are joined in $\overrightarrow{\mathbb{M}}(\mathfrak{q})=(\mathbb{A}(\mathfrak{q}), \overrightarrow{\mathbb{H}}(\mathfrak{q}))$ if there exist a fuzzy directed path $\vec{P}_{(\mathfrak{q} ;(x, y))}$ in $\overrightarrow{\mathbb{R}}(\mathfrak{q})$. The membership and non-membership degrees of directed edges in $\overrightarrow{\mathbb{M}}(\mathfrak{q})$ are evaluated as

$\left\{\begin{array}{l}\mu_{\overrightarrow{\mathbb{H}}(\mathfrak{q})}(x, y) \\ =\min \left\{\mu_{\overrightarrow{\mathbb{B}}(\mathfrak{q})}(u, v) \mid(u, v) \text { is an } \operatorname{arc} \text { of } \vec{P}^{m}(\mathfrak{q} ;(x, y))\right\} \\ v_{\overrightarrow{\mathbb{H}}(\mathfrak{q})}(x, y) \\ =\max \left\{v_{\overrightarrow{\mathbb{B}}(\mathfrak{q})}(u, v) \mid(u, v) \text { is an } \operatorname{arc} \text { of } \vec{P}^{m}(\mathfrak{q} ;(x, y))\right\},\end{array}\right.$

where $0 \leq \mu_{\overrightarrow{\mathbb{H}}(\mathfrak{q})}^{2}(x, y)+v_{\overrightarrow{\mathbb{H}}(\mathfrak{q})}^{2}(x, y) \leq 1$, for all $x, y \in X$ and $\mathfrak{q} \in Q$. 

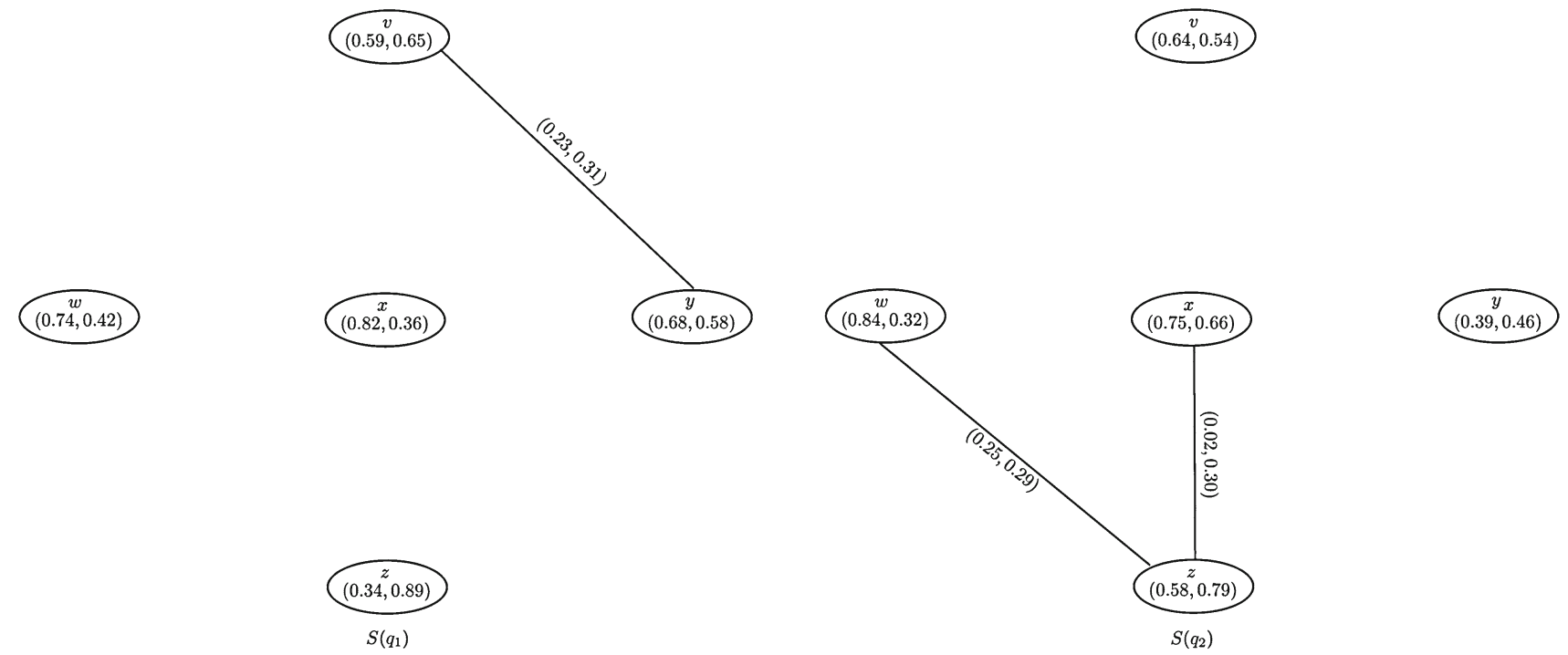

Fig. $5 \mathrm{~A} \mathrm{PFS}_{f}$ 0.3-CG
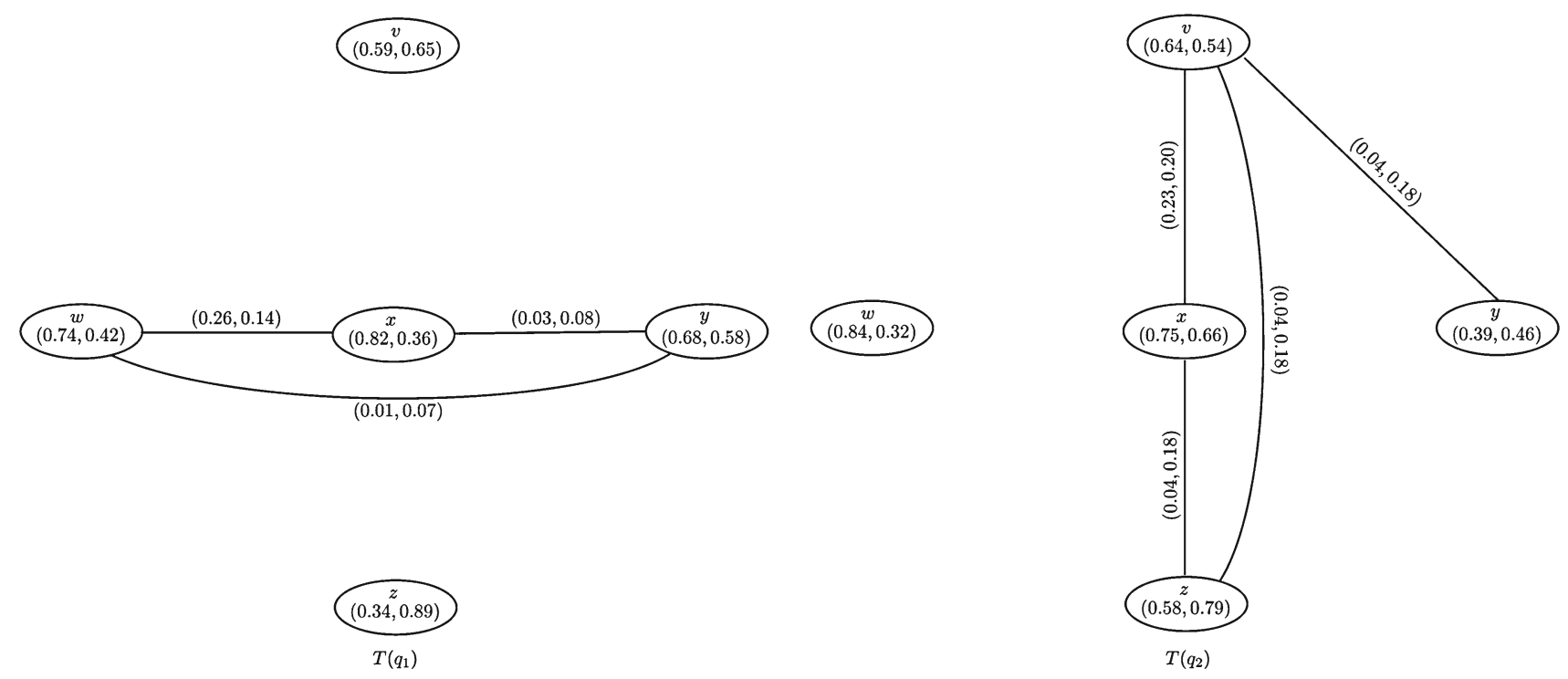

Fig. $6 \mathrm{~A} \mathrm{PFS}_{f}$ 0.5-economic CG

Example 7 Consider the $\mathrm{PFS}_{f} \mathrm{G} \overrightarrow{\mathbb{G}}=(\mathbb{A}, \overrightarrow{\mathbb{B}}, Q)$ of Example 2. We can construct its 2-step $\mathrm{PFS}_{f}$ digraph $\overrightarrow{\mathbb{G}}_{2}=$ $(\mathbb{A}, \overrightarrow{\mathbb{H}}, Q)$ as shown in Fig. 7 with

$$
\begin{aligned}
\overrightarrow{\mathbb{M}}\left(\mathfrak{q}_{1}\right)= & \left(\mathbb{A}\left(\mathfrak{q}_{1}\right), \overrightarrow{\mathbb{H}}\left(\mathfrak{q}_{1}\right)\right)=(\{\langle v,(0.59,0.65)\rangle, \\
& \langle w,(0.74,0.42)\rangle,\langle x,(0.82,0.36)\rangle, \\
& \langle y,(0.68,0.58)\rangle,\langle z,(0.34,0.89)\rangle\}, \\
\{\langle(v, w),(0.55,0.57)\rangle,\langle(v, x),(0.55,0.49)\rangle, & \\
& \langle(v, z),(0.31,0.82)\rangle, \\
& \langle(y, w),(0.28,0.82)\rangle\}), \\
\overrightarrow{\mathbb{M}}\left(\mathfrak{q}_{2}\right)= & \left(\mathbb{A}\left(\mathfrak{q}_{2}\right), \overrightarrow{\mathbb{H}}\left(\mathfrak{q}_{2}\right)\right)=(\{\langle v,(0.64,0.54)\rangle,
\end{aligned}
$$

$$
\begin{aligned}
& \langle w,(0.84,0.32)\rangle,\langle x,(0.75,0.66)\rangle, \\
& \langle y,(0.39,0.46)\rangle,\langle z,(0.58,0.79)\rangle\}, \\
& \{\langle(w, v),(0.57,0.72)\rangle,\langle(w, x),(0.57,0.73)\rangle, \\
& \quad\langle(w, y),(0.33,0.72)\rangle,\langle(z, v),(0.58,0.73)\rangle, \\
& \quad\langle(z, y),(0.38,0.73)\rangle\}) .
\end{aligned}
$$

Definition 17 An m-step PFS $_{f}$ out-neighborhood $\mathbb{N}_{m}^{+}(x)$ of a vertex $x$ of a $\operatorname{PFS}_{f}$ digraph $\overrightarrow{\mathbb{G}}$ is a $\operatorname{PFS}_{f} S \mathbb{N}_{m}^{+}(x)=$ $\left\{\left\langle\mathfrak{q}, \mathbb{N}_{m}^{+}(\mathfrak{q})(x)\right\rangle \quad \mid \quad \forall \mathfrak{q} \quad \in \quad Q\right\} \quad$ such that $\mathbb{N}_{m}^{+}(\mathfrak{q})$ $(x)=\left\{\left\langle y,\left(\mu_{\overrightarrow{\mathbb{B}}(\mathfrak{q})}(y), \nu_{\overrightarrow{\mathbb{B}}(\mathfrak{q})}(y)\right)\right\rangle \mid \vec{P}^{m}{ }_{(\mathfrak{q} ;(x, y))}\right.$ exists $\}$

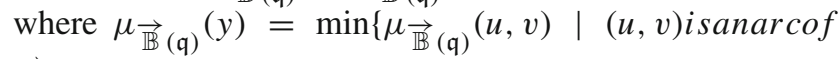
$\left.\vec{P}^{m}{ }_{(\mathfrak{q} ;(x, y))}\right\}$ and $\nu_{\overrightarrow{\mathbb{B}}(\mathfrak{q})}(y)=\max \left\{\nu_{\overrightarrow{\mathbb{B}}(\mathfrak{q})}(u, v) \mid(u, v)\right.$ is 

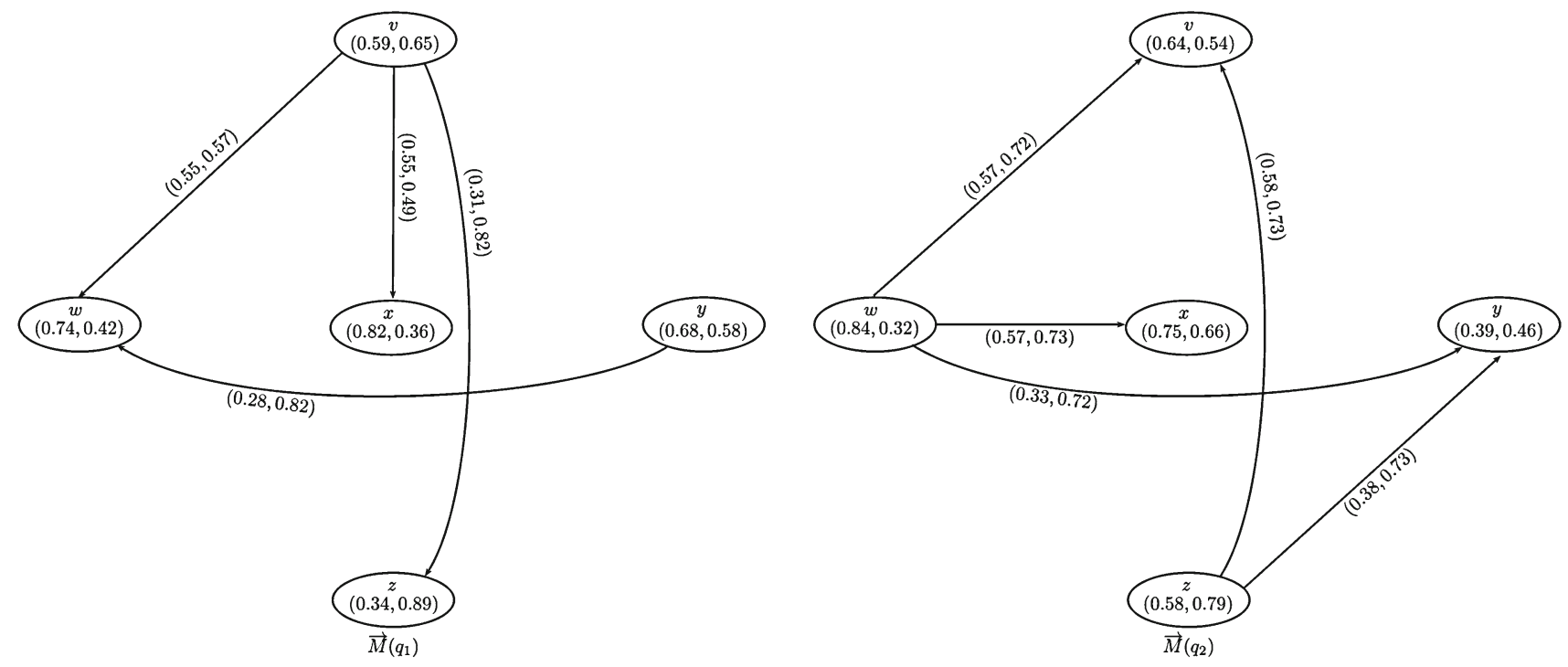

Fig. 7 A 2-step PFS $_{f}$ digraph

an arc of $\left.\vec{P}_{(\mathfrak{q} ;(x, y))}\right\}$. An $m$-step $\mathrm{PFS}_{f}$ in-neighborhood

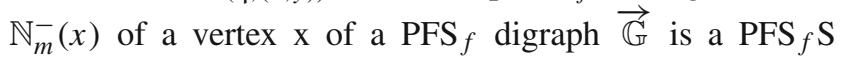
$\mathbb{N}_{m}^{-}(x)=\left\{\left\langle\mathfrak{q}, \mathbb{N}_{m}^{-}(\mathfrak{q})(x)\right\rangle \mid \forall \mathfrak{q} \in Q\right\}$ such that $\mathbb{N}_{m}^{-}(\mathfrak{q})(x)=$ $\left\{\left\langle y,\left(\mu_{\overrightarrow{\mathbb{B}}_{(\mathfrak{q})}}(y), \nu_{\overrightarrow{\mathbb{B}}_{(\mathfrak{q})}}(y)\right)\right\rangle \quad \mid \vec{P}^{m}(\mathfrak{q} ;(y, x))\right.$ exists $\}$ where $\mu_{\overrightarrow{\mathbb{B}}(\mathfrak{q})}(y)=\min \left\{\mu_{\overrightarrow{\mathbb{B}}(\mathfrak{q})}(u, v) \mid(u, v)\right.$ isanarcof

$\left.\vec{P}^{m}{ }_{(\mathfrak{q} ;(y, x))}\right\}$ and $v_{\overrightarrow{\mathbb{B}}(\mathfrak{q})}(y)=\max \left\{v_{\overrightarrow{\mathbb{B}}(\mathfrak{q})}(u, v) \mid(u, v)\right.$ isanarc of $\left.\vec{P}_{(\mathfrak{q} ;(y, x))}^{m}\right\}$

Example 8 Consider again the $\mathrm{PFS}_{f}$ digraph $\overrightarrow{\mathbb{G}}=(\mathbb{A}, \overrightarrow{\mathbb{B}}, Q)$, displayed in Fig. 2.

The $\mathrm{PFS}_{f}$ 2-step out-neighborhoods of vertices of $\overrightarrow{\mathbb{G}}$ are

$$
\begin{aligned}
& \mathbb{N}_{2}^{-}(x)=\left\{\frac{\{\langle v,(0.55,0.49)\rangle\}}{\mathfrak{q}_{1}}, \frac{\{\langle w,(0.57,0.73)\rangle\}}{\mathfrak{q}_{2}}\right\}, \\
& \mathbb{N}_{2}^{-}(y)=\left\{\frac{\{\}}{\mathfrak{q}_{1}}, \frac{\{\langle w,(0.33,0.72)\rangle,\langle z,(0.38,0.73)\rangle\}}{\mathfrak{q}_{2}}\right\}, \\
& \mathbb{N}_{2}^{-}(z)=\left\{\frac{\{\langle v,(0.31,0.82)\rangle\}}{\mathfrak{q}_{1}}, \frac{\{\}}{\mathfrak{q}_{2}}\right\} .
\end{aligned}
$$

Definition 18 An m-step Pythagorean fuzzy soft competition graph ( $m$-step $\left.\operatorname{PFS}_{f} C G\right) \mathbb{C}_{m}(\mathbb{G})=(\mathbb{A}, \mathbb{D}, Q)$ of a $\mathrm{PFS}_{f}$ digraph $\overrightarrow{\mathbb{G}}=(\mathbb{A}, \overrightarrow{\mathbb{B}}, Q)$ is an undirected $\mathrm{PFS}_{f} \mathrm{G}$ which has the same $\operatorname{PFS}_{f}$ vertex set as in $\overrightarrow{\mathbb{G}}$ and for all $\mathfrak{q} \in Q$, the Pythagorean fuzzy points $x, y \in X$ are joined in $\mathbb{S}(\mathfrak{q})=$

$$
\begin{aligned}
\mathbb{N}_{2}^{+}(v) & =\left\{\frac{\{\langle w,(0.55,0.57)\rangle,\langle x,(0.55,0.49)\rangle,\langle z,(0.31,0.82)\rangle\}}{\mathfrak{q}_{1}}, \frac{\{\}}{\mathfrak{q}_{2}}\right\}, \\
\mathbb{N}_{2}^{+}(w) & =\left\{\frac{\{\}}{\mathfrak{q}_{1}}, \frac{\{\langle v,(0.57,0.72)\rangle,\langle x,(0.57,0.73)\rangle,\langle y,(0.33,0.72)\rangle\}}{\mathfrak{q}_{2}}\right\}, \\
\mathbb{N}_{2}^{+}(x) & =\left\{\frac{\{\}}{\mathfrak{q}_{1}}, \frac{\{\}}{\mathfrak{q}_{2}}\right\}, \\
\mathbb{N}_{2}^{+}(y) & =\left\{\frac{\{\langle w,(0.28,0.82)\rangle\}}{\mathfrak{q}_{1}}, \frac{\{\}}{\mathfrak{q}_{2}}\right\}, \\
\mathbb{N}_{2}^{+}(z) & =\left\{\frac{\{\}}{\mathfrak{q}_{1}}, \frac{\{\langle v,(0.58,0.73)\rangle,\langle y,(0.38,0.73)\rangle\}}{\mathfrak{q}_{2}}\right\} .
\end{aligned}
$$

The $\mathrm{PFS}_{f}$ 2-step in-neighborhoods of vertices of $\overrightarrow{\mathbb{G}}$ are

$$
\begin{aligned}
\mathbb{N}_{2}^{-}(v) & =\left\{\frac{\{\}}{\mathfrak{q}_{1}}, \frac{\{\langle w,(0.57,0.72)\rangle,\langle z,(0.58,0.73)\rangle\}}{\mathfrak{q}_{2}}\right\}, \\
\mathbb{N}_{2}^{-}(w) & =\left\{\frac{\{\langle v,(0.55,0.57)\rangle,\langle y,(0.28,0.82)\rangle\}}{\mathfrak{q}_{1}}, \frac{\{\}}{\mathfrak{q}_{2}}\right\},
\end{aligned}
$$

$(\mathbb{A}(\mathfrak{q}), \mathbb{D}(\mathfrak{q}))$ if and only if $\mathbb{N}_{m}^{+}(\mathfrak{q})(x) \cap \mathbb{N}_{m}^{+}(\mathfrak{q})(y)$ is nonempty set. The membership as well as non-membership grade of edges in $\mathbb{S}(\mathfrak{q})$ are evaluated as follows:

$\left\{\begin{array}{l}\mu_{\mathbb{D}(\mathfrak{q})}(x, y)=\left(\mu_{\mathbb{A}(\mathfrak{q})} \wedge \mu_{\mathbb{A}(\mathfrak{q})}\right) h_{\mu}\left(\mathbb{N}_{m}^{+}(\mathfrak{q})(x) \cap \mathbb{N}_{m}^{+}(\mathfrak{q})(y)\right), \\ \nu_{\mathbb{D}(\mathfrak{q})}(x, y)=\left(v_{\mathbb{A}(\mathfrak{q})} \vee v_{\mathbb{A}(\mathfrak{q})}\right) h_{v}\left(\mathbb{N}_{m}^{+}(\mathfrak{q})(x) \cap \mathbb{N}_{m}^{+}(\mathfrak{q})(y)\right),\end{array}\right.$ 
such that $0 \leq \mu_{\mathbb{D}(\mathfrak{q})}^{2}(x, y)+v_{\mathbb{D}(\mathfrak{q})}^{2}(x, y) \leq 1$, for all $x, y \in X$ and $\mathfrak{q} \in Q$.

Example 9 The 2-step $\mathrm{FS}_{f} \mathrm{CG} \mathbb{C}_{2}(\overrightarrow{\mathbb{G}})=(\mathbb{A}, \mathbb{D}, Q)$, for the $\operatorname{PFS}_{f} \mathrm{G} \overrightarrow{\mathbb{G}}=(\mathbb{A}, \overrightarrow{\mathbb{B}}, Q)$ of Example 2, is shown in Fig. 8 .

The edge membership values can be calculated as

$$
\begin{aligned}
& \mathbb{N}_{2}^{+}\left(\mathfrak{q}_{1}\right)(v) \cap \mathbb{N}_{2}^{+}\left(\mathfrak{q}_{1}\right)(y) \\
& =\{\langle w,(0.28,0.82)\rangle\} \\
& \Rightarrow \quad h\left(\mathbb{N}_{2}^{+}\left(\mathfrak{q}_{1}\right)(v) \cap \mathbb{N}_{2}^{+}\left(\mathfrak{q}_{1}\right)(y)\right)=(0.28,0.82) \\
& \left\{\begin{aligned}
\mu_{\mathbb{D}\left(\mathfrak{q}_{1}\right)}(v, y)=\left(\mu_{\mathbb{A}\left(\mathfrak{q}_{1}\right)}(v) \wedge \mu_{\mathbb{A}\left(\mathfrak{q}_{1}\right)}(y)\right) h_{\mu}\left(\mathbb{N}_{2}^{+}\left(\mathfrak{q}_{1}\right)(v)\right. \\
\left.\cap \mathbb{N}_{2}^{+}\left(\mathfrak{q}_{1}\right)(y)\right)=(0.59 \wedge 0.68)(0.25) \\
=0.16 \\
v_{\mathbb{D}\left(\mathfrak{q}_{1}\right)}(v, y) \\
=\left(v_{\mathbb{A}\left(\mathfrak{q}_{1}\right)}(v) \vee v_{\mathbb{A}\left(\mathfrak{q}_{1}\right)}(y)\right) h_{v}\left(\mathbb{N}_{2}^{+}\left(\mathfrak{q}_{1}\right)(v)\right. \\
\left.\cap \mathbb{N}_{2}^{+}\left(\mathfrak{q}_{1}\right)(y)\right)=(0.65 \vee 0.58)(0.82) \\
=0.53,
\end{aligned}\right.
\end{aligned}
$$

such that $\mu_{\mathbb{D}\left(\mathfrak{q}_{1}\right)}^{2}(v, w)+v_{\mathbb{D}\left(\mathfrak{q}_{1}\right)}^{2}(v, w) \leq 1$. Thus, we have $\mathbb{C}_{2}(\mathbb{G})=\left(\mathbb{S}\left(\mathfrak{q}_{1}\right), \mathbb{S}\left(\mathfrak{q}_{2}\right)\right)$, where

$$
\begin{aligned}
\mathbb{S}\left(\mathfrak{q}_{1}\right)= & \left(\mathbb{A}\left(\mathfrak{q}_{1}\right), \mathbb{D}\left(\mathfrak{q}_{1}\right)\right)=(\{\langle v,(0.59,0.65)\rangle, \\
& \langle w,(0.74,0.42)\rangle,\langle x,(0.82,0.36)\rangle, \\
& \langle y,(0.68,0.58)\rangle,\langle z,(0.34,0.89)\rangle\}, \\
\{ & \langle(v, y),(0.16,0.53)\rangle\}), \\
\mathbb{S}\left(\mathfrak{q}_{2}\right)= & \left(\mathbb{A}\left(\mathfrak{q}_{2}\right), \mathbb{D}\left(\mathfrak{q}_{2}\right)\right)=(\{\langle v,(0.64,0.54)\rangle, \\
& \langle w,(0.84,0.32)\rangle,\langle x,(0.75,0.66)\rangle, \\
& \langle y,(0.39,0.46)\rangle,\langle z,(0.58,0.79)\rangle\}, \\
& \{\langle(w, z),(0.33,0.58)\rangle\}) .
\end{aligned}
$$

Definition 19 An $m$-step Pythagorean fuzzy soft economic competition graph $\left(m\right.$-step $\left.\operatorname{PFS}_{f} E C G\right) \mathbb{E}_{m}(\mathbb{G})=(\mathbb{A}, \mathbb{F}, Q)$ of a $\operatorname{PFS}_{f}$ digraph $\overrightarrow{\mathbb{G}}=(\mathbb{A}, \overrightarrow{\mathbb{B}}, Q)$ is an undirected $\mathrm{PFS}_{f} \mathrm{G}$ which has the same PFS $f$ vertex set as in $\overrightarrow{\mathbb{G}}$ and for all $\mathfrak{q} \in Q$, the Pythagorean fuzzy points $x, y \in X$ are joined in $\mathbb{T}(\mathfrak{q})=(\mathbb{A}(\mathfrak{q}), \mathbb{F}(\mathfrak{q}))$ if and only if $\mathbb{N}_{m}^{-}(\mathfrak{q})(x) \cap \mathbb{N}_{m}^{-}(\mathfrak{q})(y)$ is non-empty set. The membership as well as non-membership grade of edges in $\mathbb{T}(\mathfrak{q})$ are evaluated as follows:

$\left\{\begin{array}{l}\mu_{\mathbb{F}(\mathfrak{q})}(x, y)=\left(\mu_{\mathbb{A}(\mathfrak{q})} \wedge \mu_{\mathbb{A}(\mathfrak{q})}\right) h_{\mu}\left(\mathbb{N}_{m}^{-}(\mathfrak{q})(x) \cap \mathbb{N}_{m}^{-}(\mathfrak{q})(y)\right), \\ \nu_{\mathbb{F}(\mathfrak{q})}(x, y)=\left(v_{\mathbb{A}(\mathfrak{q})} \vee v_{\mathbb{A}(\mathfrak{q})}\right) h_{v}\left(\mathbb{N}_{m}^{-}(\mathfrak{q})(x) \cap \mathbb{N}_{m}^{-}(\mathfrak{q})(y)\right),\end{array}\right.$

such that $0 \leq \mu_{\mathbb{F}(\mathfrak{q})}^{2}(x, y)+v_{\mathbb{F}(\mathfrak{q})}^{2}(x, y) \leq 1$, for all $x, y \in X$ and $\mathfrak{q} \in Q$.

Example 10 The 2-step $\operatorname{PFS}_{f} \mathrm{ECG}_{2}(\overrightarrow{\mathbb{G}})=(\mathbb{A}, \mathbb{F}, Q)$, for the $\operatorname{PFS}_{f} \mathrm{G} \overrightarrow{\mathbb{G}}=(\mathbb{A}, \overrightarrow{\mathbb{B}}, Q)$ of Example 2, is presented in Fig. 9.
The edge membership values can be calculated as

$$
\begin{aligned}
& \mathbb{N}_{2}^{-}\left(\mathfrak{q}_{1}\right)(w) \cap \mathbb{N}_{2}^{-}\left(\mathfrak{q}_{1}\right)(x) \\
& =\{\langle v,(0.55,0.57)\rangle\} \\
& \Rightarrow \quad h\left(\mathbb{N}_{2}^{-}\left(\mathfrak{q}_{1}\right)(w) \cap \mathbb{N}_{2}^{-}\left(\mathfrak{q}_{1}\right)(x)\right)=(0.55,0.57), \\
& \left\{\begin{aligned}
\mu_{\mathbb{F}\left(\mathfrak{q}_{1}\right)}(w, x) & =\left(\mu_{\mathbb{A}\left(\mathfrak{q}_{1}\right)}(w) \wedge \mu_{\mathbb{A}\left(\mathfrak{q}_{1}\right)}(x)\right) h_{\mu}\left(\mathbb{N}_{2}^{-}\left(\mathfrak{q}_{1}\right)(w)\right. \\
& \left.\cap \mathbb{N}_{2}^{-}\left(\mathfrak{q}_{1}\right)(x)\right)=(0.74 \wedge 0.82)(0.55) \\
& =0.41, \\
v_{\mathbb{F}\left(\mathfrak{q}_{1}\right)}(w, x) & =\left(v_{\mathbb{A}\left(\mathfrak{q}_{1}\right)}(w) \vee v_{\mathbb{A}\left(\mathfrak{q}_{1}\right)}(x)\right) h_{v}\left(\mathbb{N}_{2}^{-}\left(\mathfrak{q}_{1}\right)(w)\right. \\
& \left.\cap \mathbb{N}_{2}^{-}\left(\mathfrak{q}_{1}\right)(x)\right)=(0.42 \vee 0.36)(0.57) \\
& =0.24,
\end{aligned}\right.
\end{aligned}
$$

such that $\mu_{\mathbb{F}\left(\mathfrak{q}_{1}\right)}^{2}(x, y)+v_{\mathbb{F}\left(\mathfrak{q}_{1}\right)}^{2}(x, y) \leq 1$. Thus, we have $\mathbb{E}_{2}(\mathbb{G})=\left(\mathbb{T}\left(\mathfrak{q}_{1}\right), \mathbb{T}\left(\mathfrak{q}_{2}\right)\right)$, where

$$
\begin{aligned}
& \mathbb{T}\left(\mathfrak{q}_{1}\right)=\left(\mathbb{A}\left(\mathfrak{q}_{1}\right), \mathbb{F}\left(\mathfrak{q}_{1}\right)\right)=(\{\langle v,(0.59,0.65)\rangle, \\
&\langle w,(0.74,0.42)\rangle,\langle x,(0.82,0.36)\rangle, \\
&\langle y,(0.68,0.58)\rangle,\langle z,(0.34,0.89)\rangle\}, \\
&\{\langle(w, x),(0.41,0.24)\rangle,\langle(w, z),(0.10,0.73)\rangle, \\
&\quad\langle(x, z),(0.10,0.73)\rangle\}), \\
& \mathbb{T}\left(\mathfrak{q}_{2}\right)=\left(\mathbb{A}\left(\mathfrak{q}_{2}\right), \mathbb{F}\left(\mathfrak{q}_{2}\right)\right)=(\{\langle v,(0.64,0.54)\rangle, \\
& \quad\langle w,(0.84,0.32)\rangle,\langle x,(0.75,0.66)\rangle, \\
&\langle y,(0.39,0.46)\rangle,\langle z,(0.58,0.79)\rangle\}, \\
&\langle(v, x),(0.36,0.48)\rangle,\langle(v, y),(0.15,0.39)\rangle, \\
&\langle(x, y),(0.13,0.48)\rangle\}) .
\end{aligned}
$$

Theorem 5 Let $\overrightarrow{\mathbb{G}}=(\mathbb{A}, \overrightarrow{\mathbb{B}}, Q)$ be a PFS digraph on $X$. If $m>|X|$, then $\mathbb{C}_{m}(\overrightarrow{\mathbb{G}})=(\mathbb{A}, \mathbb{D}, Q)$ as well as $\mathbb{E}_{m}(\overrightarrow{\mathbb{G}})=$ $(\mathbb{A}, \mathbb{F}, Q)$ has no edge.

Proof Let $\overrightarrow{\mathbb{R}}(\mathfrak{q})=(\mathbb{A}(\mathfrak{q}), \overrightarrow{\mathbb{B}}(\mathfrak{q}))$ be a fuzzy sub-digraph in $\overrightarrow{\mathbb{G}}$ and $\mathbb{S}(\mathfrak{q})=(\mathbb{A}(\mathfrak{q}), \mathbb{D}(\mathfrak{q}))$ be the corresponding PFCG in $\mathbb{C}_{m}(\overrightarrow{\mathbb{G}})=(\mathbb{A}, \mathbb{D}, Q)$. If $m>|X|$, then for all $\mathfrak{q}$, it is obvious that there exist no directed path of length $m$ in $\overrightarrow{\mathbb{R}}(\mathfrak{q})$, i.e., $\mathbb{N}_{m}^{+}(\mathfrak{q})(x)=\{\}$ for all $x \in X$. Consequently, $\mathbb{N}_{m}^{+}(\mathfrak{q})(x) \cap$ $\mathbb{N}_{m}^{+}(\mathfrak{q})(y)=\{\}$ and hence $\mu_{\mathbb{D}(\mathfrak{q})}(x, y)=0=\nu_{\mathbb{D}(\mathfrak{q})}(x, y)$, $\forall x, y \in X$. As $\mathbb{S}(\mathfrak{q})$ has no edge and $\mathfrak{q}$ is arbitrary, it is clear that $\mathbb{C}_{m}(\overrightarrow{\mathbb{G}})$ is also void of edges.

The proof for the result of $\mathbb{E}_{m}(\overrightarrow{\mathbb{G}})$ is similar to the above.

Theorem 6 If $\overrightarrow{\mathbb{G}}$ is a PFS $f$ digraph and $\overrightarrow{\mathbb{G}} m$ is the $m$-step $P F S_{f}$ digraph of $\overrightarrow{\mathbb{G}}$, then

1. $\mathbb{C}_{m}(\stackrel{\overrightarrow{\mathbb{G}}}{\rightarrow})=\mathbb{C}(\stackrel{\overrightarrow{\mathbb{G}}}{\rightarrow})$,

2. $\mathbb{E}_{m}(\overrightarrow{\mathbb{G}})=\mathbb{E}\left(\overrightarrow{\mathbb{G}}_{m}\right)$. 

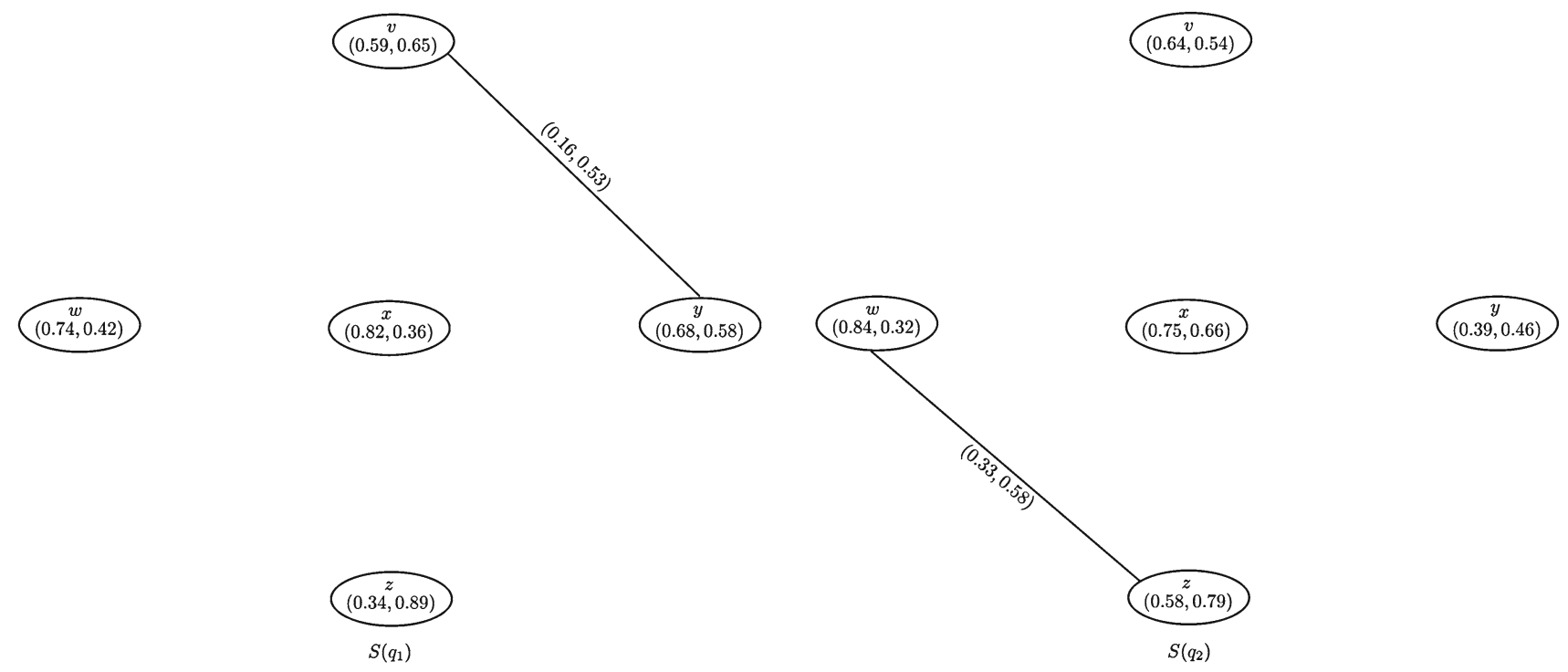

Fig. 8 A 2-step PFS $_{f} \mathrm{CG}$
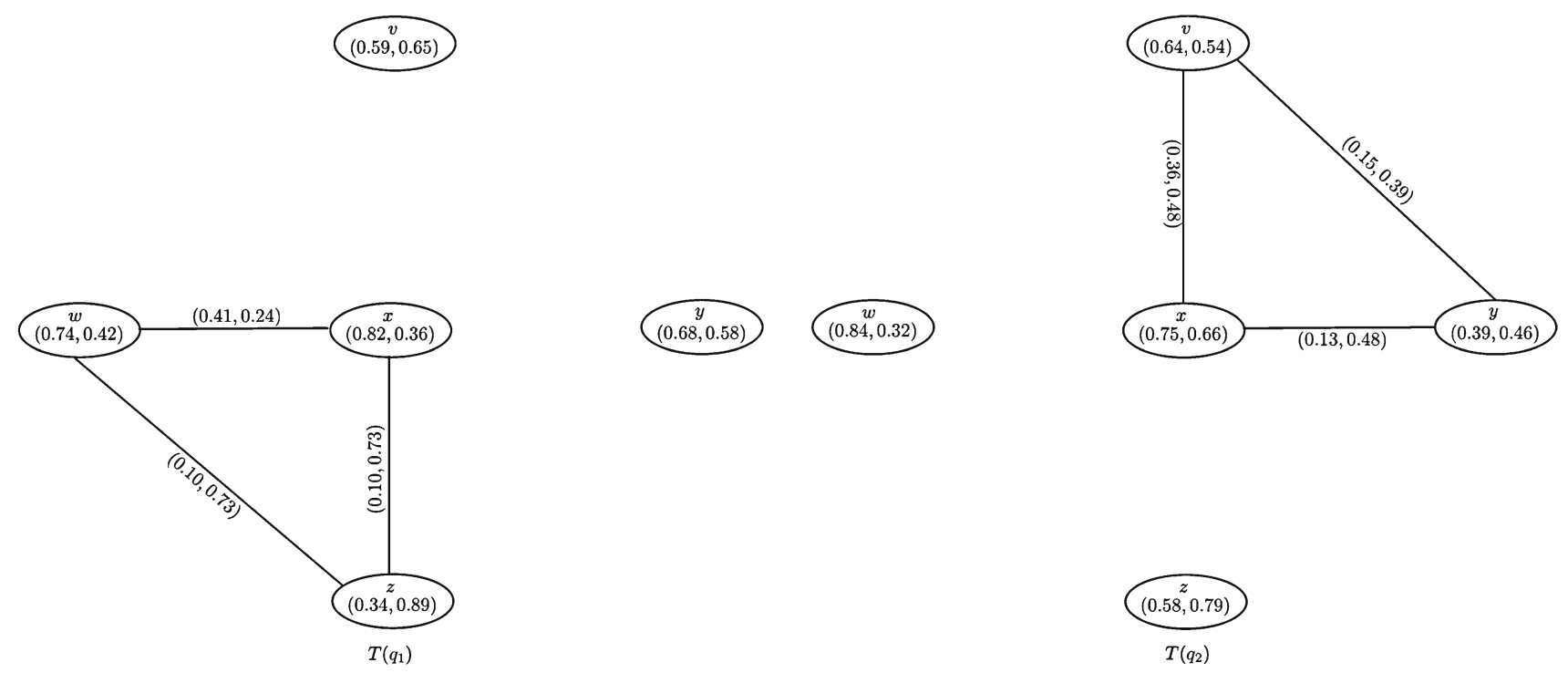

Fig. 9 A 2-step PFS $_{f}$ ECG

Theorem 7 If $\overrightarrow{\mathbb{G}^{\prime}}$ is a PFS sub-digraph of $\overrightarrow{\mathbb{G}}$, then

1. $\mathbb{C}_{m}\left(\overrightarrow{\mathbb{G}^{\prime}}\right) \subset \mathbb{C}_{m}(\overrightarrow{\mathbb{G}})$,

2. $\mathbb{E}_{m}\left(\overrightarrow{\mathbb{G}^{\prime}}\right) \subset \mathbb{E}_{m}(\overrightarrow{\mathbb{G}})$.

Proof Let $\overrightarrow{\mathbb{G}^{\prime}}=\left(\mathbb{A}^{\prime}, \overrightarrow{\mathbb{B}^{\prime}}, Q\right)$ be a PFS $f$ sub-digraph of $\overrightarrow{\mathbb{G}}=(\mathbb{A}, \overrightarrow{\mathbb{B}}, Q)$. Then $\mu_{\mathbb{A}^{\prime}(\mathfrak{q})}(x) \leq \mu_{\mathbb{A}(\mathfrak{q})}(x)$ as well as $v_{\mathbb{A}^{\prime}(\mathfrak{q})}(x) \geq v_{\mathbb{A}(\mathfrak{q})}(x)$ and $\mu_{\mathbb{B}^{\prime}(\mathfrak{q})}(x, y) \leq \mu_{\mathbb{B}(\mathfrak{q})}(x, y)$ as well as $v_{\mathbb{B}^{\prime}(\mathfrak{q})}(x, y) \geq v_{\mathbb{B}(\mathfrak{q})}(x, y)$ for each $x, y \in X$ and $\mathfrak{q} \in Q$.

To prove the statement (2), consider the $m$-step PFS PCG $_{f}$ $\mathbb{E}_{m}\left(\overrightarrow{\mathbb{G}^{\prime}}\right)=\left(\mathbb{A}^{\prime}, \mathbb{D}^{\prime}, Q\right)$ and $\mathbb{E}_{m}(\overrightarrow{\mathbb{G}})=(\mathbb{A}, \mathbb{D}, Q)$ of $\overrightarrow{\mathbb{G}}^{\prime}$ and $\overrightarrow{\mathbb{G}}$, respectively. As the vertex set of a digraph and its corresponding CG is same so are the $\mathrm{PFS}_{f}$ vertex sets of $\overrightarrow{\mathbb{G}}$ and $\mathbb{E}_{m}(\overrightarrow{\mathbb{G}})$ as well as $\overrightarrow{\mathbb{G}^{\prime}}$ and $\mathbb{E}_{m}\left(\overrightarrow{\mathbb{G}^{\prime}}\right)$. This shows that $m$-step $\mathrm{PFS}_{f}$ in-neighborhood of any vertex $x$ in $\mathbb{A}^{\prime}$ is contained in the $m$-step $\mathrm{PFS}_{f}$ in-neighborhood of the same vertex in $\mathbb{A}$. So for any $x, y \in X, \mathbb{N}_{m}^{\prime}(x) \cap \mathbb{N}_{m}^{\prime-}(y)$ in $\overrightarrow{\mathbb{G}^{\prime}}$ is a PFS $f$ subset of that in $\overrightarrow{\mathbb{G}}$. In other words, for each $\mathfrak{q} \in Q, \mathbb{N}_{m}^{\prime-}(\mathfrak{q})(x) \cap \mathbb{N}_{m}^{\prime-}(\mathfrak{q})(y)$ in $\overrightarrow{\mathbb{G}^{\prime}}$ is a Pythagorean fuzzy subset of that in $\overrightarrow{\mathbb{G}}$, i.e., $\mu_{\mathbb{D}^{\prime}(\mathfrak{q})}(x, y) \leq \mu_{\mathbb{D}(\mathfrak{q})}(x, y)$ and $\nu_{\mathbb{D}^{\prime}(\mathfrak{q})}(x, y) \geq v_{\mathbb{D}(\mathfrak{q})}(x, y)$. Consequently, $\mathbb{E}_{m}\left(\overrightarrow{\mathbb{G}^{\prime}}\right) \subset$ $\mathbb{E}_{m}(\overrightarrow{\mathbb{G}})$.

Definition 20 Let $\overrightarrow{\mathbb{G}}=(\mathbb{A}, \overrightarrow{\mathbb{B}}, Q)$ be a $\mathrm{PFS}_{f}$ digraph on $X$. Let $s$ be a common Pythagorean fuzzy prey of 
$m$-step Pythagorean fuzzy out-neighborhoods of vertices $x_{1}, x_{2}, \ldots, x_{t}$ in Pythagorean fuzzy sub-digraph $\overrightarrow{\mathbb{R}}(\mathfrak{q})=$ $(\mathbb{A}(\mathfrak{q}), \overrightarrow{\mathbb{B}}(\mathfrak{q}))$ of $\overrightarrow{\mathbb{G}}$ such that $\mu_{\overrightarrow{\mathbb{B}}(\mathfrak{q})}\left(u_{1}, v_{1}\right), \mu_{\overrightarrow{\mathbb{B}}(\mathfrak{q})}\left(u_{2}, v_{2}\right)$, $\ldots, \mu_{\vec{B}(\mathfrak{q})}\left(u_{t}, v_{t}\right)$ be the smallest membership degree and $\nu_{\vec{B}_{(\mathfrak{q})}}\left(u_{1}, v_{1}\right), \nu_{\vec{B}_{(\mathfrak{q})}}\left(u_{2}, v_{2}\right), \ldots, \nu_{\mathbb{B}_{(\mathfrak{q})}}\left(u_{t}, v_{t}\right)$ be the largest non-

membership degree of edges in the Pythagorean fuzzy directed paths $\vec{P}_{\left(\mathfrak{q} ;\left(x_{1}, s\right)\right)}^{m}, \vec{P}_{\left(\mathfrak{q} ;\left(x_{2}, s\right)\right)}^{m}, \ldots, \vec{P}_{\left(\mathfrak{q} ;\left(x_{t}, s\right)\right)}^{m}$, respectively. We call the $m$-step Pythagorean fuzzy prey $s \in X$, a strong Pythagorean fuzzy prey in $\overrightarrow{\mathbb{R}}(\mathfrak{q})$ if for all $1 \leq i \leq t$, $\mu_{\mathbb{B}_{(\mathfrak{q})}}\left(u_{i}, v_{i}\right)>0.5$ and $\nu_{\mathbb{B}_{(\mathfrak{q})}}\left(u_{i}, v_{i}\right)<0.5$. If these condition are satisfied in $\overrightarrow{\mathbb{R}}(\mathfrak{q})$ for all $\mathfrak{q}$, then $s$ is termed as a strong $\mathrm{PFS}_{f}$ prey.

The strength of a $\mathrm{PFS}_{f}$ prey $s$ is denoted by $\operatorname{Str}(s)=$ $\left(\operatorname{Str}(s)_{\mu}, \operatorname{Str}(s)_{\nu}\right)=\left(\min _{\mathfrak{q}}\left\{\operatorname{Str}(\mathfrak{q} ; s)_{\mu}\right\}, \max _{\mathfrak{q}}\left\{\operatorname{Str}(\mathfrak{q} ; s)_{\nu}\right\}\right)$, where the mappings $\operatorname{Str}(\mathfrak{q} ; s)_{\mu}: X \rightarrow[0,1]$ and $\operatorname{Str}(\mathfrak{q} ; s)_{\nu}:$ $X \rightarrow[0,1]$ are defined as

$$
\left\{\begin{array}{l}
\operatorname{Str}(\mathfrak{q} ; s)_{\mu}=\frac{\sum_{i=1}^{t} \mu \vec{B}_{(\mathfrak{q})}\left(u_{i}, v_{i}\right)}{t} \\
\operatorname{Str}(\mathfrak{q} ; s)_{\nu}=\frac{\sum_{i=1}^{t} \nu_{\vec{B}(\mathfrak{q})}\left(u_{i}, v_{i}\right)}{t}
\end{array}\right.
$$

Definition 21 Let $\overrightarrow{\mathbb{G}}=(\mathbb{A}, \overrightarrow{\mathbb{B}}, Q)$ be a $\mathrm{PFS}_{f}$ digraph on $X$. Let $b$ be a common Pythagorean fuzzy buyer of $m$ step fuzzy in-neighborhoods of vertices $x_{1}, x_{2}, \ldots, x_{t}$ in Pythagorean fuzzy sub-digraph $\overrightarrow{\mathbb{R}}(\mathfrak{q})=(\mathbb{A}(\mathfrak{q}), \overrightarrow{\mathbb{B}}(\mathfrak{q}))$ of $\overrightarrow{\mathbb{G}}$ such that $\mu_{\mathbb{B}_{(\mathfrak{q})}}\left(u_{1}, v_{1}\right), \mu_{\overrightarrow{\mathbb{B}}(\mathfrak{q})}\left(u_{2}, v_{2}\right), \ldots, \mu_{\vec{B}_{(\mathfrak{q})}}\left(u_{t}, v_{t}\right)$ be the smallest membership degree and $v_{\vec{B}(\mathfrak{q})}\left(u_{1}, v_{1}\right)$, $v_{\vec{B}_{(\mathfrak{q})}}\left(u_{2}, v_{2}\right), \ldots, v_{\vec{B}(\mathfrak{q})}\left(u_{t}, v_{t}\right)$ be the largest non -membership degree of edges in the Pythagorean fuzzy directed paths $\vec{P}_{\left(\mathfrak{q} ;\left(b, x_{1}\right)\right)}^{m}, \vec{P}_{\left(\mathfrak{q} ;\left(b, x_{2}\right)\right)}^{m}, \ldots, \vec{P}_{\left(\mathfrak{q} ;\left(b, x_{t}\right)\right)}^{m}$, respectively. We call the $m$-step Pythagorean fuzzy buyer $b \in X$, a strong Pythagorean fuzzy buyer in $\overrightarrow{\mathbb{R}}(\mathfrak{q})$ if for all $1 \leq i \leq t$, $\mu_{\vec{B}(\mathfrak{q})}\left(u_{i}, v_{i}\right)>0.5$ and $\nu_{\vec{B}_{(\mathfrak{q})}}\left(u_{i}, v_{i}\right)<0.5$. If these condition are satisfied in $\overrightarrow{\mathbb{R}}(\mathfrak{q})$ for all $\mathfrak{q}$, then $b$ is termed as a strong $\mathrm{PFS}_{f}$ buyer.

The strength of $a \operatorname{PFS}_{f}$ buyer $b$ is denoted by $\operatorname{Str}(b)=$ $\left(\operatorname{Str}(b)_{\mu}, \operatorname{Str}(b)_{\nu}\right)=\left(\min _{\mathfrak{q}}\left\{\operatorname{Str}(\mathfrak{q} ; b)_{\mu}\right\}, \max _{\mathfrak{q}}\left\{\operatorname{Str}(\mathfrak{q} ; b)_{\nu}\right\}\right)$, where the mappings $\operatorname{Str}(\mathfrak{q} ; b)_{\mu}: X \rightarrow[0,1]$ and $\operatorname{Str}(\mathfrak{q} ; b)_{\nu}:$ $X \rightarrow[0,1]$ are defined as

$$
\left\{\begin{array}{l}
\operatorname{Str}(\mathfrak{q} ; b)_{\mu}=\frac{\sum_{i=1}^{t} \mu \vec{B}_{(\mathfrak{q})}\left(u_{i}, v_{i}\right)}{t} \\
\operatorname{Str}(\mathfrak{q} ; b)_{v}=\frac{\sum_{i=1}^{t} v_{\vec{B}(\mathfrak{q})}\left(u_{i}, v_{i}\right)}{t}
\end{array}\right.
$$

Example 11 Consider the $\mathrm{PFS}_{f}$ digraph $\overrightarrow{\mathbb{G}}=(\mathbb{A}, \overrightarrow{\mathbb{B}}, Q)$ displayed in Fig. 2.
Note that $y$ is a common Pythagorean fuzzy prey of 2step Pythagorean fuzzy out-neighborhoods of vertices $v$ and $z$ in $\overrightarrow{\mathbb{R}}\left(\mathfrak{q}_{1}\right)$ as well as $\overrightarrow{\mathbb{R}}\left(\mathfrak{q}_{2}\right)$, and its strength is $\operatorname{Str}(y)=$ $(0.35,0.71)$. Similarly, $w$ is a common Pythagorean fuzzy buyer of 2-step Pythagorean fuzzy in-neighborhoods of vertices $x$ and $z$ in $\overrightarrow{\mathbb{R}}\left(\mathfrak{q}_{1}\right)$ as well as $\overrightarrow{\mathbb{R}}\left(\mathfrak{q}_{2}\right)$, and its strength is $\operatorname{Str}(w)=(0.47,0.69)$.

Theorem 8 Let $\overrightarrow{\mathbb{G}}=(\mathbb{A}, \overrightarrow{\mathbb{B}}, Q)$ be a PFS digraph on $a$ non-void set $X$.

1. If a PFS $f$ prey $s$ of $\overrightarrow{\mathbb{G}}$ is independent strong then the strength of $s$ satisfies $\operatorname{Str}(s)_{\mu}>0.5$ and $\operatorname{Str}(b)_{\nu}<0.5$.

2. If a PFS $f$ buyer $b$ of $\overrightarrow{\mathbb{G}}$ is independent strong then the strength of $b$ satisfies $\operatorname{Str}(b)_{\mu}>0.5$ and $\operatorname{Str}(b)_{\nu}<0.5$.

Proof Let $s$ be a common $\mathrm{PFS}_{f}$ vertex of $m$-step $\mathrm{PFS}_{f}$ outneighborhoods of $\mathrm{PFS}_{f}$ vertices $x_{1}, x_{2}, \ldots, x_{t}$, i.e., $s$ is a common Pythagorean fuzzy vertex of $m$-step Pythagorean fuzzy out-neighborhoods of Pythagorean fuzzy vertices $x_{1}, x_{2}, \ldots, x_{t}$ in Pythagorean fuzzy sub-digraph $\overrightarrow{\mathbb{R}}(\mathfrak{q})=$ $(\mathbb{A}(\mathfrak{q}), \overrightarrow{\mathbb{B}}(\mathfrak{q}))$ of $\overrightarrow{\mathbb{G}}$, for all $\mathfrak{q}$. This means that there exist the Pythagorean fuzzy directed paths $\vec{P}_{\left(\mathfrak{q} ;\left(x_{1}, s\right)\right)}^{m}$, $\vec{P}_{\left(\mathfrak{q} ;\left(x_{2}, s\right)\right)}^{m}, \ldots, \vec{P}_{\left(\mathfrak{q} ;\left(x_{t}, s\right)\right)}^{m}$ of length $m$ in $\overrightarrow{\mathbb{R}}(\mathfrak{q}), \forall \mathfrak{q}$. Suppose $\mu_{\overrightarrow{\mathbb{B}}(\mathfrak{q})}\left(u_{1}, v_{1}\right), \mu_{\overrightarrow{\mathbb{B}}_{(\mathfrak{q})}}\left(u_{2}, v_{2}\right), \ldots, \mu_{\overrightarrow{\mathbb{B}}(\mathfrak{q})}\left(u_{t}, v_{t}\right)$ be the smallest membership degree and $\overrightarrow{\mathbb{B}}_{(\mathfrak{q})}$ $\left(u_{1}, v_{1}\right), \nu_{\overrightarrow{\mathbb{B}}(\mathfrak{q})}\left(u_{2}, v_{2}\right), \ldots, v_{\mathbb{B}_{(\mathfrak{q})}}\left(u_{t}, v_{t}\right)$ be the largest nonmembership degree of edges in paths $\vec{P}_{\left(\mathfrak{q} ;\left(x_{1}, s\right)\right)}^{m}, \vec{P}_{\left(\mathfrak{q} ;\left(x_{2}, s\right)\right)}^{m}$, $\ldots, \vec{P}_{\left(\mathfrak{q} ;\left(x_{t}, s\right)\right)}^{m}$, respectively. Assume that prey $s$ is independent strong in $\overrightarrow{\mathbb{R}}(\mathfrak{q})$ then the Pythagorean fuzzy directed edges fulfill the conditions $\mu_{\mathbb{B}_{(\mathfrak{q})}}\left(u_{i}, v_{i}\right)>0.5$ and $\nu_{\vec{B}(\mathfrak{q})}\left(u_{i}, v_{i}\right)$, for all $1 \leq i \leq t$. This implies

$\operatorname{Str}(\mathfrak{q} ; s)_{\mu}>\frac{0.5+0.5+\cdots+0.5(\text { ttimes })}{t}=0.5$,

$\operatorname{Str}(\mathfrak{q} ; s)_{v}<\frac{0.5+0.5+\cdots+0.5(\text { ttimes })}{t}=0.5$.

Since $\mathfrak{q}$ is arbitrary, $0.5<\min _{\mathfrak{q}} \operatorname{Str}(\mathfrak{q} ; s)_{\mu}=\operatorname{Str}(s)_{\mu}$ and $0.5>\max _{\mathfrak{q}} \operatorname{Str}(\mathfrak{q} ; s)_{v}=\operatorname{Str}(s)_{\nu}$.

The proof to statement (2) is similar.

Definition 22 For a positive integer $p$, the $p$-competition Pythagorean fuzzy soft graph ( $p$-competition $\mathrm{PFS}_{f} \mathrm{G}$ ) of a $\operatorname{PFS}_{f}$ digraph $\overrightarrow{\mathbb{G}}=(\mathbb{A}, \overrightarrow{\mathbb{B}}, Q)$ is an undirected PFSG $\mathbb{C}^{p}(\overrightarrow{\mathbb{G}})=(\mathbb{A}, \mathbb{D}, Q)$ which has the same $\mathrm{PFS}_{f}$ vertex set as in $\overrightarrow{\mathbb{G}}$ and for all $\mathfrak{q} \in Q$, the Pythagorean fuzzy points $x, y \in X$ are joined in $\mathbb{S}(\mathfrak{q})=(\mathbb{A}(\mathfrak{q}), \mathbb{D}(\mathfrak{q}))$ if and only if for each $\mathfrak{q} \in Q\left|\operatorname{supp}\left(\mathbb{N}^{+}(\mathfrak{q})(x) \cap \mathbb{N}^{+}(\mathfrak{q})(y)\right)\right| \geq p$. The membership as well as non-membership grade of edges in 


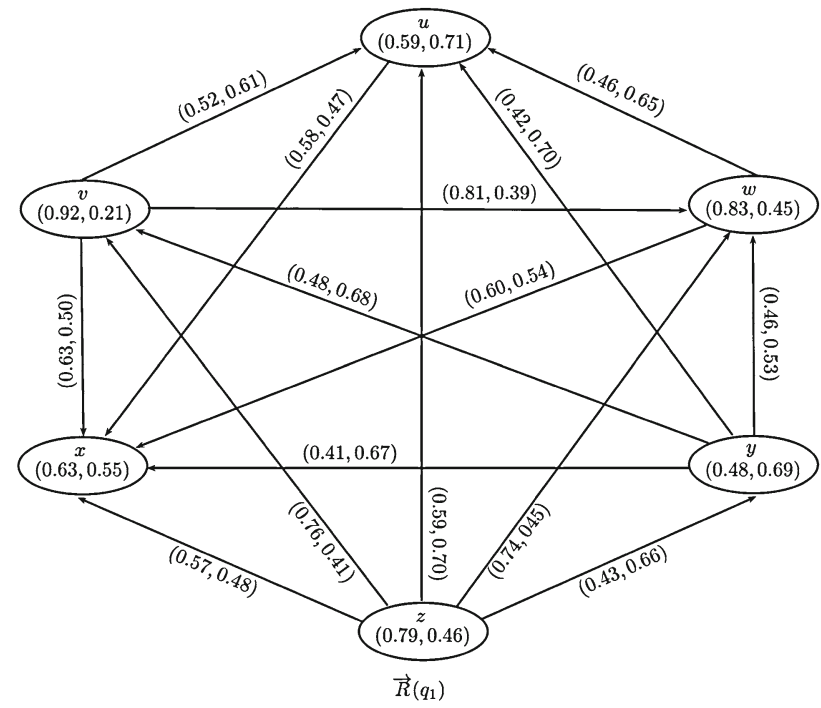

Fig. $10 \mathrm{APFS}_{f}$ digraph

$\mathbb{S}(\mathfrak{q})$ are computed as follows:

$$
\left\{\begin{array}{l}
\mu_{\mathbb{D}(\mathfrak{q})}(x, y) \\
=\frac{(n-p)+1}{n}\left(\mu_{\mathbb{A}(\mathfrak{q})}(x) \wedge \mu_{\mathbb{A}(\mathfrak{q})}(y)\right) h_{\mu}\left(\mathbb{N}^{+}(\mathfrak{q})(x)\right. \\
\left.\cap \mathbb{N}^{+}(\mathfrak{q})(y)\right), \\
v_{\mathbb{D}(\mathfrak{q})}(x, y) \\
=\frac{(n-p)+1}{n}\left(\nu_{\mathbb{A}(\mathfrak{q})}(x) \vee v_{\mathbb{A}(\mathfrak{q})}(y)\right) h_{v}\left(\mathbb{N}^{+}(\mathfrak{q})(x)\right. \\
\left.\cap \mathbb{N}^{+}(\mathfrak{q})(y)\right),
\end{array}\right.
$$

such that $0 \leq \mu_{\mathbb{D}(\mathfrak{q})}^{2}(x, y)+v_{\mathbb{D}(\mathfrak{q})}^{2}(x, y) \leq 1$, for all $x, y \in X$ and $\mathfrak{q} \in Q$, where $n=\left|\operatorname{supp}\left(\mathbb{N}^{+}(\mathfrak{q})(x) \cap \mathbb{N}^{+}(\mathfrak{q})(y)\right)\right|$.

Example 12 Let us take into consideration a $\mathrm{PFS}_{f}$ digraph $\overrightarrow{\mathbb{G}}=(\mathbb{A}, \overrightarrow{\mathbb{B}}, Q)$ presented in the Fig. 10 such that

$$
\begin{aligned}
\overrightarrow{\mathbb{R}}\left(\mathfrak{q}_{1}\right)= & \left(\mathbb{A}\left(\mathfrak{q}_{1}\right), \overrightarrow{\mathbb{B}}\left(\mathfrak{q}_{1}\right)\right)=(\{\langle u,(0.59,0.71)\rangle, \\
& \langle v,(0.92,0.21)\rangle,\langle w,(0.83,0.45)\rangle, \\
& \langle x,(0.63,0.55)\rangle,\langle y,(0.48,0.69)\rangle, \\
\langle & z,(0.79,0.46)\rangle\},\{\langle(u, x),(0.58,0.47)\rangle, \\
& \langle(v, u),(0.52,0.61)\rangle,\langle(v, w),(0.81,0.39)\rangle, \\
& \langle(v, x),(0.63,0.50)\rangle, \\
& \langle(w, u),(0.46,0.65)\rangle,\langle(w, x),(0.60,0.54)\rangle, \\
& \langle(y, u),(0.42,0.70)\rangle, \\
& \langle(y, v),(0.48,0.68)\rangle,\langle(y, w),(0.46,0.53)\rangle, \\
& \langle(y, x),(0.41,0.67)\rangle,\langle(z, u),(0.59,0.70)\rangle, \\
& \langle(z, v),(0.76,0.41)\rangle,\langle(z, w),(0.74,0.45)\rangle, \\
& \langle(z, x),(0.57,0.48)\rangle, \\
& \langle(z, y),(0.43,0.66)\rangle\}) .
\end{aligned}
$$

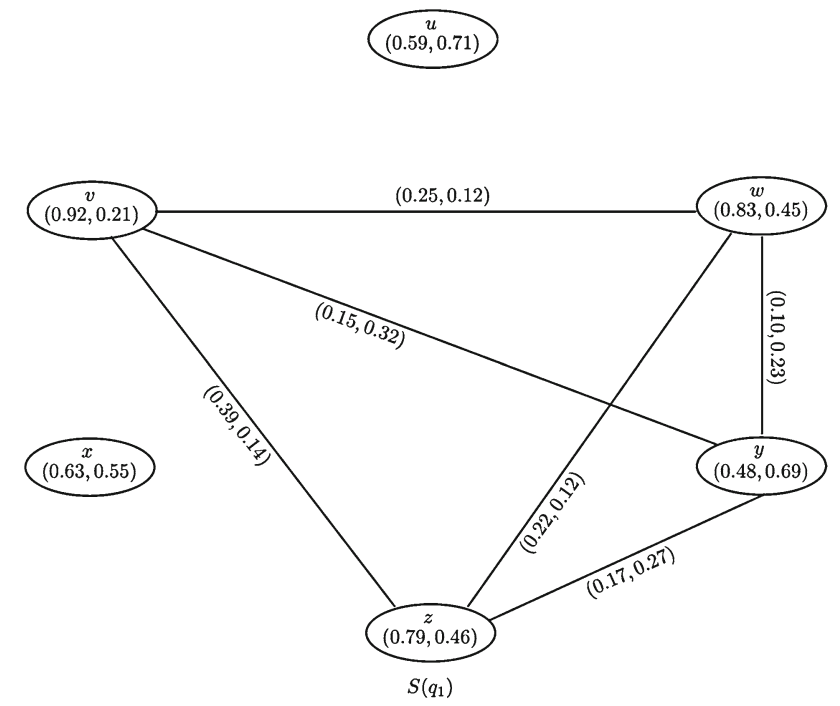

Fig. 11 A 2-competition $\mathrm{PFS}_{f} \mathrm{G}$

In order to have a 2-competition $\mathrm{PFS}_{f} \mathrm{G}$, we can compute the edge membership and non-membership degrees as

$$
\begin{aligned}
& \mathbb{N}^{+}\left(\mathfrak{q}_{1}\right)(v) \cap \mathbb{N}^{+}\left(\mathfrak{q}_{1}\right)(w) \\
& =\{\langle u,(0.46,0.65)\rangle,\langle x,(0.60,0.54)\rangle\} \\
& \Rightarrow n=\left|\operatorname{supp}\left(\mathbb{N}^{+}\left(\mathfrak{q}_{1}\right)(v) \cap \mathbb{N}^{+}\left(\mathfrak{q}_{1}\right)(w)\right)\right|=2, \\
& \left\{\begin{array}{l}
\mu_{\mathbb{D}\left(\mathfrak{q}_{1}\right)}(v, w) \\
=\frac{(n-2)+1}{n}\left(\mu_{\mathbb{A}\left(\mathfrak{q}_{1}\right)}(v) \wedge \mu_{\mathbb{A}\left(\mathfrak{q}_{1}\right)}(w)\right) h_{\mu}\left(\mathbb{N}^{+}\left(\mathfrak{q}_{1}\right)(v)\right. \\
\left.\cap \mathbb{N}^{+}\left(\mathfrak{q}_{1}\right)(w)\right)=\frac{1}{2}(0.92 \wedge 0.83)(0.60)=0.25, \\
v_{\mathbb{D}\left(\mathfrak{q}_{1}\right)}(v, w) \\
=\frac{(n-2)+1}{n}\left(v_{\mathbb{A}\left(\mathfrak{q}_{1}\right)}(v) \vee v_{\mathbb{A}\left(\mathfrak{q}_{1}\right)}(w)\right) h_{v}\left(\mathbb{N}^{+}\left(\mathfrak{q}_{1}\right)(v)\right. \\
\left.\cap \mathbb{N}^{+}\left(\mathfrak{q}_{1}\right)(w)\right)=\frac{1}{2}(0.21 \wedge 0.45)(0.54)=0.12,
\end{array}\right.
\end{aligned}
$$

where $\mu_{\mathbb{D}\left(\mathfrak{q}_{1}\right)}^{2}(u, v)+v_{\mathbb{D}\left(\mathfrak{q}_{1}\right)}^{2}(u, v) \leq 1$. The $\operatorname{PFS}_{f} \mathrm{G} \mathbb{C}^{2}(\overrightarrow{\mathbb{G}})$ obtained is given in Fig. 11.

Definition 23 For a positive integer $p$, the $p$-economic competition Pythagorean fuzzy soft graph ( $p$-economic competition $\left.\mathrm{PFS}_{f} \mathrm{G}\right)$ of a $\mathrm{PFS}_{f}$ digraph $\overrightarrow{\mathbb{G}}=(\mathbb{A}, \overrightarrow{\mathbb{B}}, Q)$ is an undirected PFSG $\mathbb{E}^{p}(\overrightarrow{\mathbb{G}})=(\mathbb{A}, \mathbb{F}, Q)$ which has the same PFS $_{f}$ vertex set as in $\overrightarrow{\mathbb{G}}$ and for all $\mathfrak{q} \in Q$, the Pythagorean fuzzy points $x, y \in X$ are joined in $\mathbb{T}(\mathfrak{q})=(\mathbb{A}(\mathfrak{q}), \mathbb{F}(\mathfrak{q}))$ if and only if for each $\mathfrak{q} \in Q\left|\operatorname{supp}\left(\mathbb{N}^{-}(\mathfrak{q})(x) \cap \mathbb{N}^{-}(\mathfrak{q})(y)\right)\right| \geq$ $p$. The membership as well as non-membership grade of 

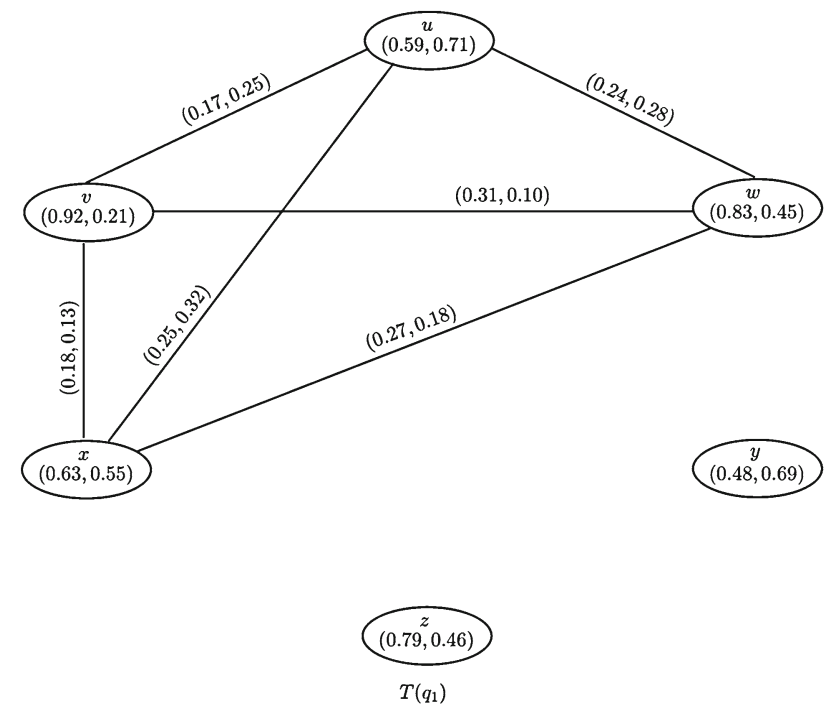

Fig. 12 A 2-economic competition $\mathrm{PFS}_{f} \mathrm{G}$

edges in $\mathbb{T}(\mathfrak{q})$ are computed as follows:

$$
\left\{\begin{array}{l}
\mu_{\mathbb{F}(\mathfrak{q})}(x, y) \\
=\frac{(n-p)+1}{n}\left(\mu_{\mathbb{A}(\mathfrak{q})}(x) \wedge \mu_{\mathbb{A}(\mathfrak{q})}(y)\right) h_{\mu}\left(\mathbb{N}^{-}(\mathfrak{q})(x)\right. \\
\left.\cap \mathbb{N}^{-}(\mathfrak{q})(y)\right), \\
v_{\mathbb{F}(\mathfrak{q})}(x, y) \\
=\frac{(n-p)+1}{n}\left(v_{\mathbb{A}(\mathfrak{q})}(x) \vee v_{\mathbb{A}(\mathfrak{q})}(y)\right) h_{\nu}\left(\mathbb{N}^{-}(\mathfrak{q})(x)\right. \\
\left.\cap \mathbb{N}^{-}(\mathfrak{q})(y)\right),
\end{array}\right.
$$

such that $0 \leq \mu_{\mathbb{F}(\mathfrak{q})}^{2}(x, y)+v_{\mathbb{F}(\mathfrak{q})}^{2}(x, y) \leq 1$, for all $x, y \in X$ and $\mathfrak{q} \in Q$, where $n=\left|\operatorname{supp}\left(\mathbb{N}^{-}(\mathfrak{q})(x) \cap \mathbb{N}^{-}(\mathfrak{q})(y)\right)\right|$.

Example 13 Consider again the $\mathrm{PFS}_{f}$ digraph $\overrightarrow{\mathbb{G}}=(\mathbb{A}, \overrightarrow{\mathbb{B}}, Q)$ of Example 12. In order to have a 2-economic competition $\mathrm{PFS}_{f} \mathrm{G}$, we can compute the edge membership and nonmembership degrees as

$$
\begin{aligned}
& \mathbb{N}^{-}\left(\mathfrak{q}_{1}\right)(u) \cap \mathbb{N}^{-}\left(\mathfrak{q}_{1}\right)(v) \\
& \quad=\{\langle y,(0.42,0.70)\rangle,\langle z,(0.59,0.70)\rangle\} \Rightarrow n \\
& =\mid \operatorname{supp}\left(\mathbb{N}^{-}\left(\mathfrak{q}_{1}\right)(u) \cap \mathbb{N}^{-}\left(\mathfrak{q}_{1}\right)(v) \mid=2,\right. \\
& \left\{\begin{array}{l}
\mu_{\mathbb{F}\left(\mathfrak{q}_{1}\right)}(u, v) \\
=\frac{(n-2)+1}{n}\left(\mu_{\mathbb{A}\left(\mathfrak{q}_{1}\right)}(u) \wedge \mu_{\mathbb{A}\left(\mathfrak{q}_{1}\right)}(v)\right) h_{\mu}\left(\mathbb{N}^{-}\left(\mathfrak{q}_{1}\right)(u)\right. \\
\left.\cap \mathbb{N}^{-}\left(\mathfrak{q}_{1}\right)(v)\right)=\frac{1}{2}(0.59 \wedge 0.92)(0.59)=0.17, \\
v_{\mathbb{F}\left(\mathfrak{q}_{1}\right)}(u, v) \\
=\frac{(n-2)+1}{n}\left(v_{\mathbb{A}\left(\mathfrak{q}_{1}\right)}(u) \vee v_{\mathbb{A}\left(\mathfrak{q}_{1}\right)}(v)\right) h_{v}\left(\mathbb{N}^{-}\left(\mathfrak{q}_{1}\right)(u)\right. \\
\left.\cap \mathbb{N}^{-}\left(\mathfrak{q}_{1}\right)(v)\right)=\frac{1}{2}(0.71 \wedge 0.21)(0.70)=0.25,
\end{array}\right.
\end{aligned}
$$

where $\mu_{\mathbb{F}\left(\mathfrak{q}_{1}\right)}^{2}(u, v)+v_{\mathbb{F}\left(\mathfrak{q}_{1}\right)}^{2}(u, v) \leq 1$. The PFS $f \mathbb{E}^{2}(\overrightarrow{\mathbb{G}})$ obtained is given in Fig. 12.

\section{Competition among forest trees}

Competition among organisms is a universal phenomenon that varies with respect to the factors involved in their development and growth. Its importance lies in the fact that severe competition among the species of a community can alter its structure. Competition inspires human beings to enhance their performance by putting more effort that brings satisfaction of doing things better. They usually compete for food, riches, prestige and mates. Animals and plants also struggle and compete with their own specie members as well as with the individuals of other species for their survival. Competition among the individuals of a specie is termed as intra-specific competition, whereas that within different species is called inter-specific competition.

Plants compete slowly which often went on for years as compared to animals. The resources that are essential for the survival and development of a plant are not necessarily available in sufficient quantity in the environment where it has to grow. There are various resources whose abundance or shortage influence the plants to compete for their survival. These are: (i) light (when one plant is shaded by nearby plants), (ii) water (when the dampness in soil is suboptimal), (iii) heat (when radiant energy is not available in cold areas), (iv) space (when the seeds sown are dense), (v) nutrients (when nutrients in soil are not ideally distributed), (vi) carbon dioxide (during vigorous photosynthesis in dense communities), and (vii) oxygen (as required by roots) [20]. Plants require nutrients throughout the year which include the macronutrients like nitrogen, potassium, phosphorus, calcium, sulfur and magnesium as well as micronutrients such as manganese, copper, iron, zinc etc., but their amounts needed are different for distinct plants. A plant can acquire nutrients from atmosphere, native soil nutrients, weathering of rocks and dropped litter of plants. Especially, nitrogen-fixing bacteria provide lots of nitrogen in soil. The amount of these resources in the environment of plants varies from season to season.

Consider the example of forest trees found in the mixed conifer forests of California. The climate of California changes to a great extent from burning hot to freezing cold. Some mixed conifer forests of California are found on the mountains of Sierra Nevada. In these mountains, the climate at the beginning of year is freezing cold which gradually changes to a pleasant weather during the early months of summer, hot during the summer season and then again turns to snowy winter at the end. During winter season in mixed conifer forests, the plants vigorously require water, sunlight and heat. Winter in these forests is so white that leaves and limbs of some trees cannot bear the snow load and thus fall while the other trees that have flexible branches shed heavy snow and thus survive. However, they require more space and light in summer because of regrowth of new limbs and leaves which steal the light as well as space of the relatively 
small or the new growing trees. Moreover, the plants that are shade-intolerant like Ponderosa pine which die in the constant absence of light require sunlight in all seasons. Similarly, white fir which is drought-intolerant need sufficient water in each season for its survival and development [15].

Consider a set $\{$ Ponderosa pine, Sugar pine, White fir, Incense cedar, Douglas-fir, Red fir, Lodgepole pine, Jaffrey pine $\}$ of forest trees and a set \{Water, Nutrients, Sunlight, Heat, Space $\}$ of resources that are required for the development of the considered trees. Also, consider a set $\left\{\mathfrak{q}_{1}, \mathfrak{q}_{2}\right\}$ of attributes where $\mathfrak{q}_{1}$ symbolizes winter season and $\mathfrak{q}_{2}$ symbolizes the summer season. A PFS $f$ digraph that presents the forest trees and their interaction with resources is given in Fig. 13. With regards to the considered parameter (season), the membership as well as non-membership grade of (a) trees represent their growth and growth inhibition, (b) resources denote the resource availability and shortage, and (c) directed edges narrates the accessability and inaccessability of resource to tree, in that season. For example, in $\overrightarrow{\mathbb{R}}\left(\mathfrak{q}_{1}\right)$, the directed edge from the Ponderosa pine to space has degree $(0.82,0.21)$ which shows that the space available to plant is $67.24 \%$, not available is $4.41 \%$, and the hesitation is $28.35 \%$. Table 2 represents the $\mathrm{PFS}_{f}$ out-neighborhoods of forest trees for growth resources.

The competition among trees for resources relative to winter (by $\mathbb{S}\left(\mathfrak{q}_{1}\right)$ ) and summer (by $\mathbb{S}\left(\mathfrak{q}_{2}\right)$ ) seasons is shown in Fig. 14 as a $\mathrm{PFS}_{f}$ CG. The edge joining Ponderosa pine and Lodgepole pine has degree $(0.4290,0.0630)$ in $\mathbb{S}\left(\mathfrak{q}_{1}\right)$ which narrates that they are competing (for sunlight, heat as well as space) with strength $18.4 \%$ and are not involved in competition $0.4 \%$. Table 3 gives the membership and nonmembership values of PFS $f$ edges of $\mathrm{PFS}_{f} \mathrm{CG}$. Note that the Pythagorean fuzzy subgraph $\mathbb{S}\left(\mathfrak{q}_{1}\right)$ is more dense than that of $\mathbb{S}\left(\mathfrak{q}_{2}\right)$. It is because of the fact that the resources are more depleted in winter as compared to summer, so those plants which are not tolerant to deficiency of a resource will not be able to survive and may pass by death.

The Algorithm 41 presents the procedure of the construction of $\mathrm{PFS}_{f} \mathrm{CG}$ for the $\mathrm{PFS}_{f}$ digraph. It takes the $\mathrm{PFS}_{f}$ digraph and for each of its parameter, computes the $\operatorname{PFS}_{f}$ out-neighborhoods corresponding to each tree. The heights of intersecting vertex neighborhoods and the vertex degrees give rise to the strength of competing and non-competing interactions among the forest trees.

\section{Comparative analysis}

The $\mathrm{PFS}_{f} \mathrm{~S}$ proposed by Peng et al. [27] is very assistive in order to deal with the parameterized family of PFSs. The competition in problems that have parameterized Pythagorean uncertainty should be dealt with PFS ${ }_{f}$ CGs; oth-

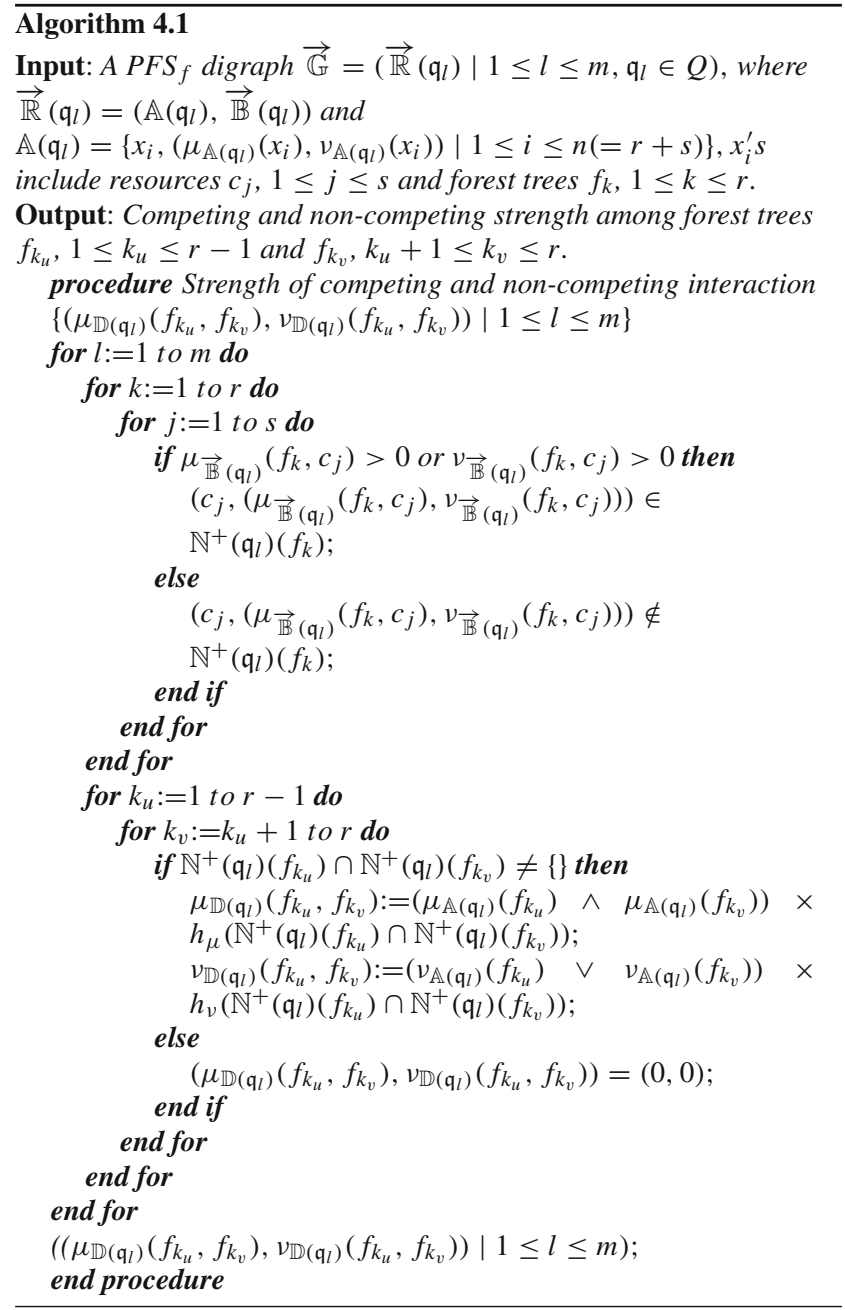

erwise, the competing and non-competing interactions could be inappropriate. We consider the PFG to present a comparison with the above discussed example of plant competition. Consider a PFS \{〈Water, $(0.63,0.41)\rangle$, 〈Nutrients, $(0.58$, $0.37)\rangle$, 〈Sunlight, $(0.73,0.25)\rangle,\langle$ Heat, $(0.32,0.86)\rangle$, 〈Space, $(0.87,0.24)\rangle\}$ of resources available for the growth of forest trees given by the PFS $\{\langle$ Ponderosapine, $(0.45,0.59)\rangle$, $\langle$ Whitefir, (0.77, 0.38) $\rangle,\langle$ Douglas - fir, $(0.82,0.29)\rangle$, $\langle$ Lodgepolepine, $(0.59,0.53)\rangle,\langle$ Jaffreypine, $(0.74,0.34)\rangle$,

$\langle$ Redfir, $(0.64,0.27)\rangle, \quad\langle$ Incensecedar, $(0.55,0.63)\rangle$

$\langle$ Sugarpine, $(0.62,0.38)\rangle\}$.

The interaction of trees with the available resources is given by a set of Pythagorean fuzzy directed edges $\{\langle($ Douglas - fir, Water $),(0.58,0.40)\rangle,\langle($ Whitefir, Nutrients $)$, $(0.57,0.36)\rangle,\langle($ Ponderosapine, Sunlight $),(0.44,0.52)\rangle$,

$\langle($ Sugarpine, Water $),(0.61,0.26)\rangle,\langle($ Sugarpine, Heat $),(0.21$, $0.34)\rangle,\langle($ Jaffreypine, Space $),(0.69,0.31)\rangle$, $\langle($ Redfir, Sunlight $)$, $(0.63,0.26)\rangle,\langle($ Incensecedar, Nutrients $),(0.53,0.52)\rangle$,

$\langle($ Douglas - fir, Space $),(0.79,0.23)\rangle,\langle($ Lodgepolepine, Heat $)$, $(0.28,0.77)\rangle,\langle(J a f f r e y p i n e$, Sunlight $),(0.73,0.29)\rangle$,

$\langle($ Ponderosapine, Water $),(0.36,0.47)\rangle\}$. 
Table $2 \mathrm{PFS}_{f}$ out-neighborhoods of forest trees

\begin{tabular}{|c|c|}
\hline Forest trees & $\mathbb{N}^{+}$(Forest trees) \\
\hline \multirow{3}{*}{ Ponderosa pine } & 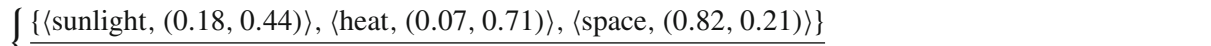 \\
\hline & 1 \\
\hline & $\{\langle$ sunlight, $(0.28,0.13)\rangle,\langle$ heat, $(0.41,0.23)\rangle\}\}$ \\
\hline \multirow{3}{*}{ Sugar pine } & $\mathfrak{q}_{2}$ \\
\hline & $\{\{\langle$ water, $(0.45,0.53)\rangle,\langle$ nutrients, $(0.43,0.47)\rangle\} \quad\{\langle$ nutrients, $(0.71,0.31)\rangle,\langle$ sunlight, $(0.82,0.09)\rangle\}$ \\
\hline & $\mathfrak{q}_{1}$ \\
\hline \multirow{2}{*}{ White fir } & $\{\langle$ water, $(0.33,0.62)\rangle,\langle$ nutrients, $(0.36,0.22)\rangle\} \quad\{\langle$ water, $(0.34,0.36)\rangle,\langle$ nutrients, $(0.41,0.43)\rangle\}\}$ \\
\hline & $\mathfrak{q}_{1}$ \\
\hline \multirow{2}{*}{ Incense cedar } & $\{\langle\langle$ water, $(0.46,0.53)\rangle,\langle$ heat,$(0.23,0.75)\rangle,\langle$ space, $(0.70,0.36)\rangle\} \quad\{\langle$ space, $(0.30,0.42)\rangle\}\}$ \\
\hline & $\mathfrak{q}_{1}$ \\
\hline \multirow{2}{*}{ Douglas-fir } & $\{\langle\langle$ water, $(0.42,0.60)\rangle,\langle$ nutrients, $(0.50,0.41)\rangle\} \quad\{\langle$ water, $(0.63,0.43)\rangle,\langle$ space, $(0.27,0.41)\rangle\}\}$ \\
\hline & $\mathfrak{q}_{1}$ \\
\hline \multirow{2}{*}{ Red fir } & $\{\{\langle$ water, $(0.40,0.52)\rangle,\langle$ heat, $(0.21,0.74)\rangle\} \quad\{\langle$ sunight, $(0.46,0.33)\rangle,\langle$ heat, $(0.50,0.11)\rangle\}\}$ \\
\hline & $\mathfrak{q}_{1}$ \\
\hline \multirow{2}{*}{ Lodgepole pine } & $\int\{\langle$ sunlight, $(0.58,0.40)\rangle,\langle$ heat, $(0.22,0.65)\rangle,\langle$ space,$(0.65,0.21)\rangle\}$ \\
\hline & $\{\langle$ nutrients, $(0.71,0.32)\rangle,\langle$ space,$(0.24,0.33)\rangle\}\}$ \\
\hline \multirow{3}{*}{ Jaffrey pine } & $\mathfrak{q}_{2}$ \\
\hline & $\int\{\langle$ nutrients, $(0.55,0.36)\rangle,\langle$ sunlight,$(0.23,0.44)\rangle,\langle$ space,$(0.77,0.18)\rangle\} \quad\{\langle$ space, $\left.(0.17,0.43)\rangle\}\right\}$ \\
\hline & $\mathfrak{q}_{1}$ \\
\hline
\end{tabular}

Table 3 The competing and non-competing strength of forest trees for resources

\begin{tabular}{ll}
\hline Forest trees & Strength of competition \\
\hline (Ponderosa pine, Sugar pine) & $((0,0),(0.1316,0.0338))$ \\
(Ponderosa pine, Incense cedar) & $((0.4970,0.1332),(0,0))$ \\
(Ponderosa pine, Red fir) & $((0.0308,0.4292),(0.1927,0.0989))$ \\
(Ponderosa pine, Lodgepole pine) & $((0.4290,0.0630),(0,0))$ \\
(Ponderosa pine, Jaffrey pine) & $((0.6391,0.0462),(0,0))$ \\
(Sugar pine, White fir) & $((0.1332,0.3854),(0.1722,0.2279))$ \\
(Sugar pine, Incense cedar) & $((0.2025,0.2067),(0,0))$ \\
(Sugar pine, Douglas-fir) & $((0.1935,0.2162),(0,0))$ \\
(Sugar pine, Red fir) & $((0.1640,0.3074),(0.2346,0.1419))$ \\
(Sugar pine, Lodgepole pine) & $((0,0),(0.5822,0.1088))$ \\
(Sugar pine, Jaffrey pine) & $((0.1935,0.1833),(0,0))$ \\
(White fir, Incense cedar) & $((0.1221,0.5084),(0,0))$ \\
(White fir, Douglas-fir) & $((0.1332,0.3362),(0.1428,0.2279))$ \\
(White fir, Red fir) & $((0.1221,0.5084),(0,0))$ \\
(White fir, Lodgepole pine) & $((0,0),(0.1722,0.2279))$ \\
(White fir, Jaffrey pine) & $((0.1332,0.2952),(0,0))$ \\
(Incense cedar, Douglas-fir) & $((0.2142,0.2760),(0.1728,0.1680))$ \\
(Incense cedar, Red fir) & $((0.1640,0.3074),(0,0))$ \\
(Incense cedar, Lodgepole pine) & $((0.4290,0.1332),(0.1608,0.1680))$ \\
(Incense cedar, Jaffrey pine) & $((0.4970,0.1332),(0.1139,0.1720))$ \\
(Douglas-fir, Red fir) & $((0.1640,0.3480),(0,0))$ \\
(Douglas-fir, Lodgepole pine) & $((0,0),(0.1536,0.1505))$ \\
(Douglas-fir, Jaffrey pine) & $((0.2550,0.1886),(0.1088,0.1505))$ \\
(Lod fir, Lodgepole pine) & $((0.0924,0.4292),(0,0))$ \\
& $((0.4290,0.0630),(0.1326,0.1462))$ \\
&
\end{tabular}


Fig. $13 \mathrm{~A} \mathrm{PFS}_{f}$ digraph presenting the interaction among forest trees and their resources

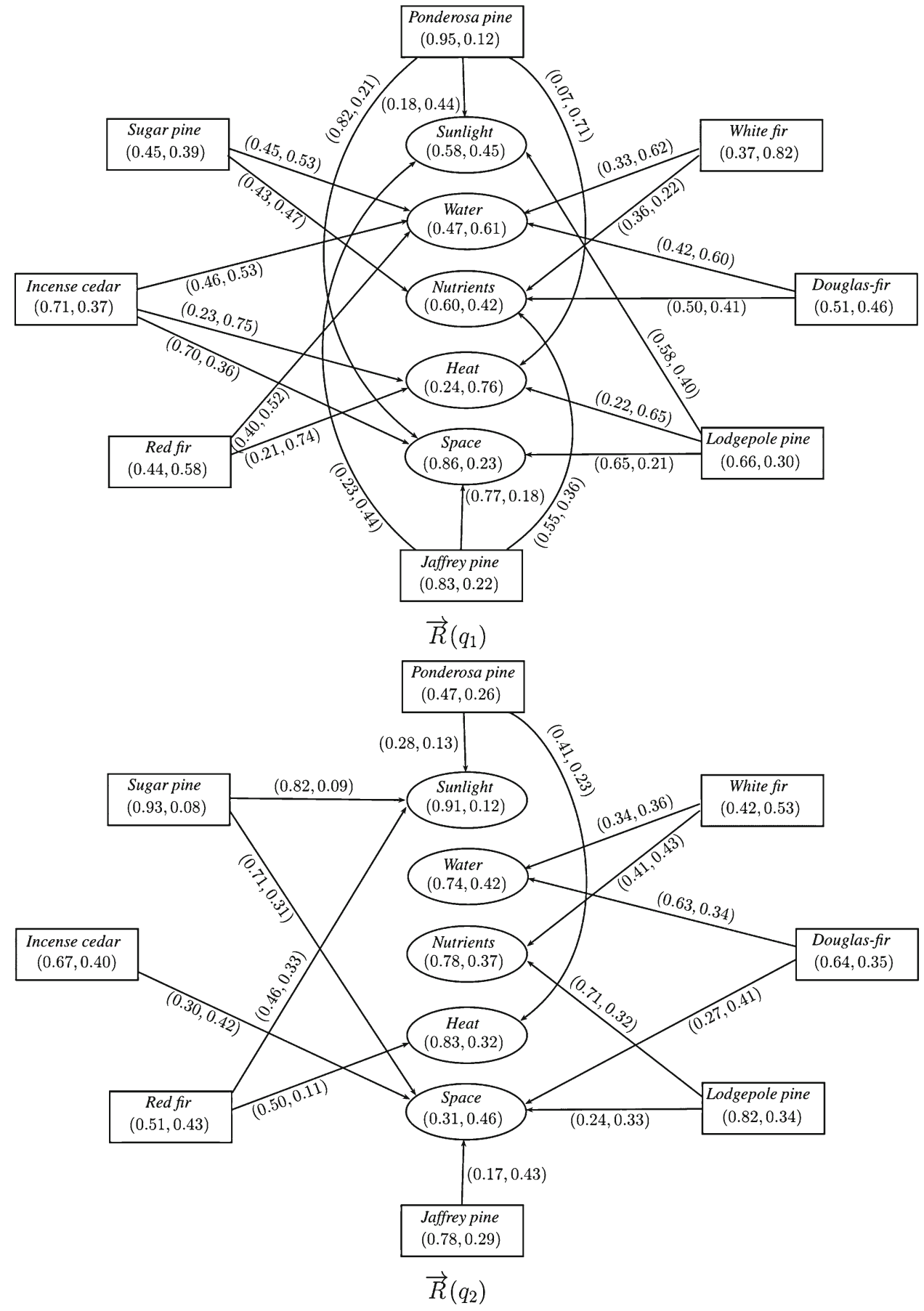

If we consider this system as to depict the annual interaction of forest trees and resources, the resulting PFCG would be incorrect. Its reason is that the resources that are responsible for the growth promotion and inhibition of plants do not remain same throughout the year in the surroundings of trees. Due to this reason, the competition among plants is not constant and varies from season to season. So, this system should be studied in the $\mathrm{PFS}_{f}$ environment so that the forest management can take measures according to each season for the growth of plants. The analysis through $\mathrm{PFS}_{f} \mathrm{CGs}$ will also be helpful to understand that the resource for which most of the trees are competing is deficient and should be provided in sufficient amounts. 
Fig. $14 \mathrm{APFS}_{f} \mathrm{CG}$

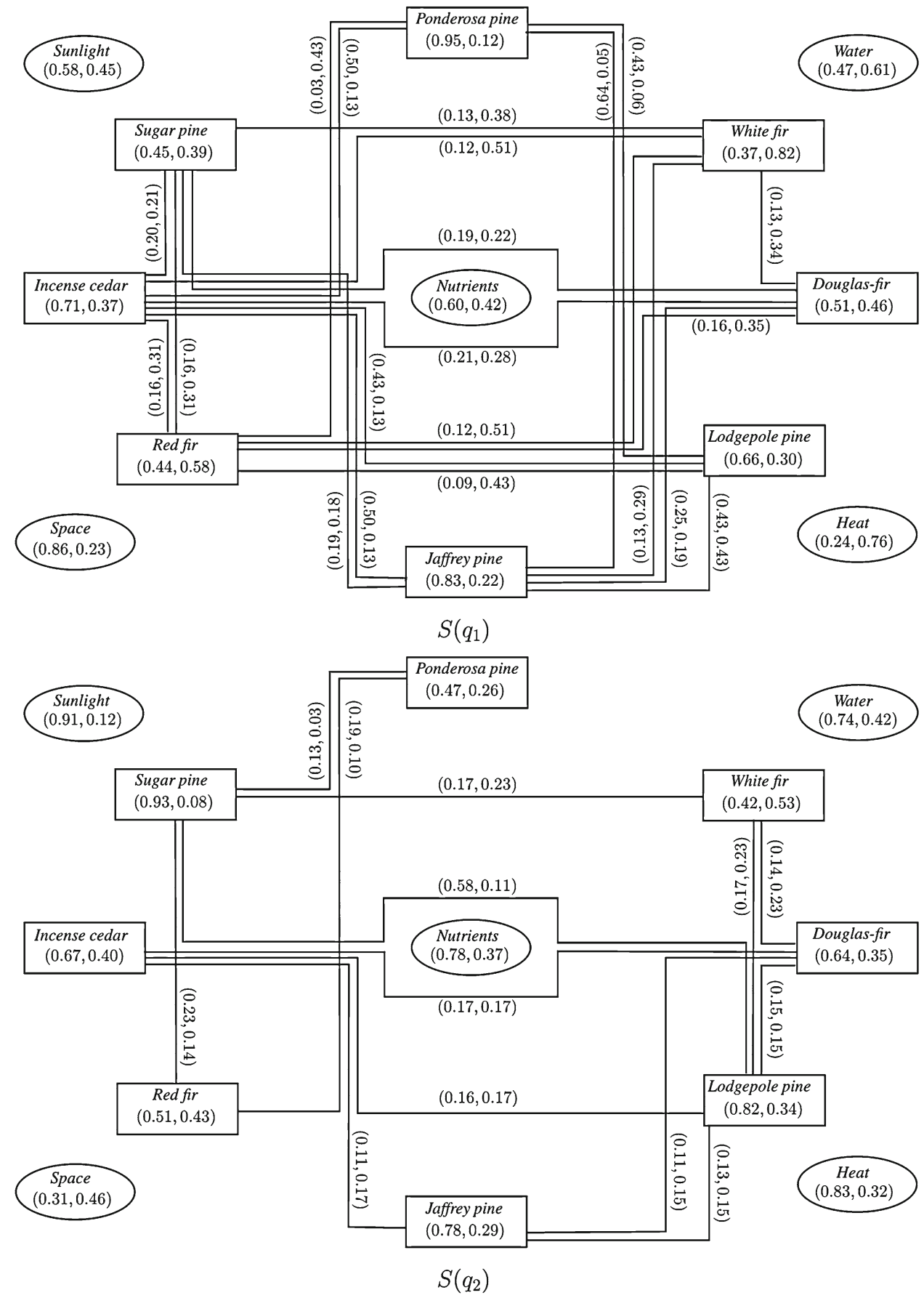

\section{Conclusion}

We often experience different types of competitions in our daily life that can be modeled as networks or graphs. The CGs of directed graphs suggested by Cohen [7] are effective to view the competing entities of system. The presence of uncertainty in actual-world problems intended the researchers to illustrate CGs in different theories. The $\mathrm{PFS}_{f}$ theory is a good blend of PFS and $S_{f} S$, which permits the assignment of Pythagorean membership values with respect to different parameters. The purpose of this article is to present competition as well as economic CGs in the $\mathrm{PFS}_{f}$ environment. Additionally, we have discussed $\mathrm{PFS}_{f} k$-CGs which are, in fact, the $\mathrm{PFS}_{f} \mathrm{CGs}$ at level $k$, where $k$ is a non-negative real number. The $m$-step $\mathrm{PFS}_{f} \mathrm{CGs}$ describe the competition among organisms for their $m$-step common preys. The edges in $p$-competition $\mathrm{PFS}_{f}$ Gs highlight all those pairwise competitors that have at least $p$-common preys. We have 
explained all these variations of $\mathrm{PFS}_{f} \mathrm{CGs}$ through examples and also provided some interesting results. All these generalizations of $\mathrm{PFS}_{f}$ CGs have significance at their own as they can represent different forms of competition in a parameter-dependent system. An example of competing forest trees for growth resources have also been discussed using $\mathrm{PFS}_{f}$ CGs. The considered set of forest trees is common in the mixed conifer forests of California. The seasonal variations are responsible for changing concentrations of resources in the environment, so it is better to study such competitions in $\mathrm{PFS}_{f}$ theory. An algorithm which calculates the strength of competing and non-competing interactions of the competing constituents of a system have also been given. The future research directions might address various topics. It is desirable to discuss (1) Pythagorean fuzzy soft competition hypergraphs, (2) Pythagorean fuzzy soft graph structure and (3) Interval-valued Pythagorean fuzzy competition graphs.

\section{Declarations}

Conflict of interest The authors declare that they have no conflict of interest regarding the publication of the research article.

Open Access This article is licensed under a Creative Commons Attribution 4.0 International License, which permits use, sharing, adaptation, distribution and reproduction in any medium or format, as long as you give appropriate credit to the original author(s) and the source, provide a link to the Creative Commons licence, and indicate if changes were made. The images or other third party material in this article are included in the article's Creative Commons licence, unless indicated otherwise in a credit line to the material. If material is not included in the article's Creative Commons licence and your intended use is not permitted by statutory regulation or exceeds the permitted use, you will need to obtain permission directly from the copyright holder. To view a copy of this licence, visit http://creativecomm ons.org/licenses/by/4.0/.

\section{References}

1. Akram M, Nawaz S (2015) On fuzzy soft graphs. Ital J Pure Appl Math 34:497-514

2. Akram M, Dudek WA, Habib A, Kenani ANA (2020) Imperfect competition models in economic market structure with q-rung picture fuzzy information. J Intell Fuzzy Syst 38:5107-5126

3. Atanassov KT (1983) Intuitionistic fuzzy sets, VII ITKR's Session, Sofia (deposed in Central Science-Technical Library of Bulgarian Academy of Science, 1697/84) (in Bulgarian)

4. Brigham RC, McMorris FR, Vitray RP (1995) Tolerance competition graphs. Linear Algebra Appl 217:41-52

5. Chen D, Tsang ECC, Wang X (2005) The parameterization reduction of soft sets and its applications. Comput Math Appl 49:757-763

6. Cho HH, Kim SR, Yunsun N (2000) The $m$-step competition graph of a digraph. Discrete Appl Math 105:115-127

7. Cohen JE (1968) Interval graphs and food webs: a finding and a problem, Document 17696-PR. RAND Corporation, Santa Monic
8. Garg H (2016) A novel correlation coefficients between Pythagorean fuzzy sets and its applications to decision-making processes. Int J Intell Syst 31:1234-1253

9. Garg H (2018) Hesitant Pythagorean fuzzy sets and their aggregation operators in multiple-attribute decision-making. Int $\mathrm{J}$ Uncertain Quantif 8:267-289

10. Garg H (2018) Linguistic Pythagorean fuzzy sets and its applications in multi attribute decision making process. Int J Intell Syst 33:1234-1263

11. Garg H (2021) New exponential operation laws and operators for interval-valued q-rung orthopair fuzzy sets in group decision making process. Neural Comput Appl. https://doi.org/10.1007/s00521021-06036-0

12. Habib A, Akram M, Farooq A (2019) q-Rung orthopair fuzzy competition graphs with application in the soil ecosystem. Mathematics 7:91-123

13. Kaufmann A (1973) Introduction a la theorie des sousensembles flous, Massonet Cie Paris

14. Kim SR, McKee TA, McMorris FR, Roberts FS (1995) pCompetition graphs. Linear Algebra Appl 217:168-178

15. Kocher SD, Harris R (2007) Tree growth and competition, Forest Stewardship series 5. Publication 8235. University of California. Agriculture and natural resources, Oakland

16. Maji PK, Biswas R, Roy AR (2001) Fuzzy soft sets. J Fuzzy Math 9:589-602

17. Maji PK, Biswas R, Roy AR (2001) Intuitionistic fuzzy soft sets. J Fuzzy Math 9:677-692

18. Maji PK, Biswas R (2003) Soft set theory. Comput Math Appl 45:555-562

19. Maji PK, Roy AR, Biswas R (2002) An application of soft sets in a decision making problem. Comput Math Appl 44:1077-1083

20. Messenger AR (1976) Root competition: grass effects on trees. J Arboric 2:228-230

21. Molodtsov D (1999) Soft set theory-first results. Comput Math Appl 37:19-31

22. Mordeson JN, Nair PS (1998) Fuzzy graphs and fuzzy hypergraphs. Physica Verlag, Heidelberg (Second Edition, 2001)

23. Mordeson JN, Mathew S, Malik DS (2018) Fuzzy graph theory with applications to human trafficking. Springer Science and Business Media LLC

24. Nawaz HS, Akram M (2021) Oligopolistic competition among the wireless internet service providers of Malaysia using fuzzy soft graphs. J Appl Math Comput. https://doi.org/10.1007/s12190021-01514-z

25. Naz S, Ashraf S, Akram M (2018) A novel approach to decisionmaking with Pythagorean fuzzy information. Mathematics 6:1-28

26. Paravathi R, Karunambigai MG (2006) Intuitionistic fuzzy graphs. In: Computational intelligence, theory and applications. Springer, Berlin, pp 139-150

27. Peng X, Yang Y, Song J, Jiang Y (2015) Pythagorean fuzzy soft set and its application. Comput Eng 41:224-229

28. Roy AR, Maji PK (2007) A fuzzy soft set theoretic approach to decision making problems. J Comput Appl Math 203:412-418

29. Sahoo S, Pal M (2016) Intuitionistic fuzzy competition graphs. J Appl Math Comput 52:37-57

30. Samanta S, Akram M, Pal M (2016) $m$-step fuzzy competition graphs. J Appl Math Comput 52:461-472

31. Samanta S, Pal M (2013) Fuzzy $k$-competition graphs and $p$ competition fuzzy graphs. Fuzzy Eng Inform 5:191-204

32. Shahzadi S, Akram M (2020) Pythagorean fuzzy soft graphs with applications. J Intell Fuzzy Syst 38:4977-4991

33. Sonntag M, Teichert HM (2004) Competition hypergraphs. Discrete Appl Math 143:324-329

34. Thumbakara RK, George B (2014) Soft graphs. Gen Math Notes $21: 75-86$ 
35. Yager RR (2013) Pythagorean fuzzy subsets. In: 2013 Joint IFSA world congress and NAFIPS annual meeting (IFSA/NAFIPS), pp $57-61$

36. Zadeh LA (1965) Fuzzy sets. Inf Control 8:338-353
Publisher's Note Springer Nature remains neutral with regard to jurisdictional claims in published maps and institutional affiliations. 